\title{
SMALL SPHERE LIMIT OF THE QUASI-LOCAL ENERGY WITH ANTI DE-SITTER SPACE REFERENCE
}

\author{
PO-NING CHEN
}

\begin{abstract}
In [13], a new quasi-local energy is introduced for spacetimes with a nonzero cosmological constant. In this article, we study the small sphere limit of this newly defined quasi-local energy for spacetimes with a negative cosmological constant. For such spacetimes, the anti de-Sitter space is used as the reference for the quasi-local energy. Given a point $p$ in a spacetime $N$, we consider a canonical family of surfaces approaching $p$ along its future null cone and evaluate the limit of the quasi-local energy. The optimal embedding equation which identifies the critical points of the quasi-local energy is solved in order to evaluate the limit. Using the optimal embedding, we show that the limit recovers the stress-energy tensor of the matter field at $p$. For vacuum spacetimes, the quasi-local energy vanishes to a higher order. In this case, the limit of the quasi-local energy is related to the Bel-Robinson tensor at $p$.
\end{abstract}

\section{INTRODUCTION}

In general relativity, a spacetime is a 4 -manifold $N$ with a Lorentzian metric $g_{\alpha \beta}$ satisfying the Einstein equation

$$
R_{\alpha \beta}-\frac{R}{2} g_{\alpha \beta}+\Lambda g_{\alpha \beta}=8 \pi T_{\alpha \beta}
$$

where $R_{\alpha \beta}$ and $R$ are the Ricci curvature and the scalar curvature of the metric $g_{\alpha \beta}$, respectively. The constant $\Lambda$ is called the cosmological constant. On the right hand side of the Einstein equation, $T_{\alpha \beta}$ is the stress-energy tensor of the matter field. For a vacuum spacetime where $T_{\alpha \beta}=0$ (which implies $R_{\alpha \beta}=\Lambda g_{\alpha \beta}$ ), the gravitational energy is typically measured by the Bel-Robinson tensor 3

$$
Q_{\mu \nu \alpha \beta}=W_{\mu \alpha}^{\rho \sigma} W_{\rho \nu \sigma \beta}+W_{\mu \beta}^{\rho \sigma} W_{\rho \nu \sigma \alpha}-\frac{1}{2} g_{\mu \nu} W_{\alpha}^{\rho \sigma \tau} W_{\beta \rho \sigma \tau},
$$

where $W_{\alpha \beta \gamma \delta}$ is the Weyl curvature tensor of the spacetime $N$. The stress-energy tensor and the Bel-Robinson tensor are useful in studying the global structure of the maximal development of the initial value problem in general relativity, see for example [4, 14].

When studying different notions of quasi-local energy, it is natural to evaluate the large sphere and the small sphere limits of the quasi-local energy to compare with the canonical measures of the gravitational energy in these situations. One expects the following [15, 29]:

The author is supported by NSF grant DMS-1308164. The author would like to thank Mu-Tao Wang and Shing-Tung Yau for helpful discussion. 
1) For a family of surfaces approaching the infinity of an isolated system (the large sphere limit), the limit of the quasi-local energy recovers the total energy-momentum of the isolated system.

2) For a family of surfaces approaching a point $p$ (the small sphere limit), the limit of the quasi-local energy recovers the stress-energy tensor in spacetimes with matter fields and the Bel-Robinson tensor for vacuum spacetimes.

For spacetimes with $\Lambda=0$, there are many works on evaluating the large sphere and the small sphere limits of different notions of quasi-local energy. See for example [5, 6, 10, 12, 18, 19, 22, 23, 24, 27, 31, 32, 33. The list we give here is by no means exhaustive. For a more comprehensive review of different notions of quasi-local energy and their limiting behaviors, see 28 and the references therein. In a sequence of papers with Wang and Yau [10, 12, 31, the above properties are confirmed for the Wang-Yau quasi-local energy. In particular, the small sphere limit of the Wang-Yau quasi-local energy is evaluated in [12] for a canonical family of surfaces approaching a point along its future null cone.

In [13], quasi-local energy and quasi-local conserved quantities are defined for spacetimes with a non-zero cosmological constant. In the same paper, the large sphere limit of the newly defined quasi-local conserved quantities is evaluated for asymptotically AdS initial data sets. It is proved that the large sphere limit of the quasi-local conserved quantities recovers the total conserved quantities for such initial data sets [1, 2, 16, 17, 20, 21]. In this article, we evaluate the small sphere limit of the new quasi-local energy and confirm the second expected property.

The construction of the quasi-local energy is based on the Hamilton-Jacobi analysis of the gravitational action using isometric embedding of the surface into the reference space as the ground state. That is, an energy is assigned to each pair of an isometric embedding of the surface into the reference space and an observer Killing field in the reference space. For $\Lambda=0$, the reference space for the Wang-Yau quasi-local energy is the Minkowski space. On the other hand, for $\Lambda<0$, the reference space is the anti de-Sitter space (AdS space). The quasi-local mass is then defined to be the minimum of the assigned quasi-local energy among all possible pair. The Euler-Lagrange equation for this energy functional is referred to as the optimal embedding equation.

To evaluate the small sphere limit of the quasi-local energy, we first study the limiting behavior of the optimal embedding equation. For the Wang-Yau quasi-local energy, the optimal embedding equation is studied in details in [9, 10, 11, 26] which played an important role in 12] for evaluating the small sphere limit. For spacetimes with a negative cosmological constant, the AdS space is used as the reference and the optimal embedding equation is more complicated for the following reasons:

1) While the existence of the isometric embedding is guaranteed by the work of 25 by Lin and Wang, the isometric embedding has to be solved explicitly to evaluate the small sphere limit. For the AdS space, the static potential is coupled to the isometric embedding equation and makes it more difficult to solve explicitly. 
2) The kernel for the optimal embedding is larger. In both [12 and this article, the optimal embedding equation for a surface in the reference space is used extensively to simplify the optimal embedding equation for the physical surface. However, due to the difference in the set of observer Killing field, the kernel of the optimal embedding equation for the AdS space is larger than that of the Minkowski space. This creates new difficulties in solving the optimal embedding equation.

Due to the above difficulties, for the AdS reference case, we can not recover all the general theorems in [9, 10, 11, 26] concerning the optimal embedding equation of the Wang-Yau quasi-local energy. Nevertheless, we are able to obtain the results necessary to evaluate the small sphere limit. For a spacetime with matter fields, there is a unique choice of the leading term of the observer killing field such that the leading term of the optimal embedding equation is solvable. However, for a vacuum spacetime, the quasi-local energy vanishes to higher order and the invertibility of the optimal embedding equation is more subtle. In fact, the leading order term of the optimal embedding equation is solvable for any choice of the observer killing field $T_{0}$. We will compute the qausi-local energy for each $T_{0}$ and the corresponding solution to the optimal embedding equation.

The structure of this article is as follows: In Section 2, we review the AdS space and its Killing fields. In Section 3, we review the quasi-local energy with reference in the AdS space. In Section 4, we describe the setting for the small sphere limit. In Section 5, we compute the expansions of the induced metric, the second fundamental forms and the connection 1-form in the small sphere limit. Using the expansions, we expend the optimal embedding equation in Section 6 and compute the non-vacuum small sphere limit of the quasi-local energy in Section 7, see Theorem [7.1. The rest of the article is devoted to the small sphere limit in vacuum spacetimes. In Section 8, for each observer Killing field, we compute the leading order term of the isometric embedding solving the leading order term of the optimal embedding equation. The isometric embedding, which depends on the choice of the observer $T_{0}$, is denoted by $Y\left(T_{0}\right)$. In the next four sections, the quasi-local energy associated to the pair $\left(Y\left(T_{0}\right), T_{0}\right)$ is computed. Section 9,10 and 11 are used to compute the three separate terms in the quasi-local energy and these results are combined in Section 12 to evaluate the limit of the quasi-local energy, see Theorem 12.1 .

\section{Anti De-Sitter SPACE And its Killing Fields}

We review the AdS space and its Killing fields in this section. Take $\mathbb{R}^{3,2}$ with the coordinate system $\left(y^{0}, y^{1}, y^{2}, y^{3}, y^{4}\right)$ and the metric

$$
-\left(d y^{4}\right)^{2}+\sum_{i=1}^{3}\left(d y^{i}\right)^{2}-\left(d y^{0}\right)^{2} .
$$

The AdS space can be identified with the hypersurface in $\mathbb{R}^{3,2}$ given by

$$
-\left(y^{4}\right)^{2}+\sum_{i=1}^{3}\left(y^{i}\right)^{2}-\left(y^{0}\right)^{2}=-\frac{1}{\kappa^{2}} .
$$


Consider the following parametrization of AdS space:

$$
\begin{aligned}
& y^{0}=\sqrt{\frac{1}{\kappa^{2}}+r^{2}} \sin t \\
& y^{1}=r \sin \theta \sin \phi \\
& y^{2}=r \sin \theta \cos \phi \\
& y^{3}=r \cos \theta \\
& y^{4}=\sqrt{\frac{1}{\kappa^{2}}+r^{2}} \cos t .
\end{aligned}
$$

This gives the static chart of the AdS space

$$
-\left(1+\kappa^{2} r^{2}\right) d t^{2}+\frac{d r^{2}}{\left(1+\kappa^{2} r^{2}\right)}+r^{2}\left(d \theta^{2}+\sin ^{2} \theta d \phi^{2}\right)
$$

and $V=\sqrt{1+\kappa^{2} r^{2}}$ be the static potential of the AdS space.

The group $S O(3,2)$ leaves this hypersurface invariant and thus the isometry group of the AdS Space is $S O(3,2)$, which is 10 dimensional. In particular, a Killing field of the AdS space can be written as

$$
\mathfrak{K}=A\left(y^{0} \frac{\partial}{\partial y^{4}}-y^{4} \frac{\partial}{\partial y^{0}}\right)-B_{i}\left(y^{0} \frac{\partial}{\partial y^{i}}+y^{i} \frac{\partial}{\partial y^{0}}\right)-C_{j}\left(y^{4} \frac{\partial}{\partial y^{j}}+y^{j} \frac{\partial}{\partial y^{4}}\right)+D_{p} \epsilon_{p q r} y^{q} \frac{\partial}{\partial y^{r}} .
$$

For simplicity, we will write $\mathfrak{K}=(A, \vec{B}, \vec{C}, \vec{D})$ and consider $\vec{B}, \vec{C}$, and $\vec{D}$ as vectors in $\mathbb{R}^{3}$. An observer Killing field $T_{0}$ is a timelike hypersurface-orthogonal Killing field such that

$$
\min -\left\langle T_{0}, T_{0}\right\rangle=1 .
$$

The observer Killing fields in the AdS space are characterized in [8, Proposition 3.1].

Proposition 2.1. A Killing field $\mathfrak{K}$ of the form (2.1) is an observer Killing field if and only if

$$
\begin{aligned}
A \vec{D} & =-\vec{B} \times \vec{C} \\
A & >\max \{|\vec{B}|,|\vec{C}|,|\vec{D}|\}
\end{aligned}
$$

and

$$
A^{2}+|\vec{D}|^{2}-|\vec{B}|^{2}-|\vec{C}|^{2}=\kappa^{2} .
$$

Remark 1. Proposition 3.1 of [8] states the above result for $\kappa=1$. It is straightforward to recover the result for general $\kappa$ from the proof.

Remark 2. In particular, for an observer Killing field, we have

$$
A \geq \sqrt{\kappa^{2}+|\vec{C}|^{2}}
$$

We will later normalize our spacetime by choosing $\kappa=1$. This corresponds to $\Lambda=-3$ in the Einstein equation. 


\section{Quasi-local energy With anti De-Sitter Reference}

In this section, we review the quasi-local energy with reference in the AdS space defined in [13. Let $\Sigma$ be a closed embedded spacelike 2-surface in a spacetime $N$. We assume the mean curvature vector $H$ of $\Sigma$ is spacelike. Let $J$ be the reflection of $H$ through the future outgoing light cone in the normal bundle of $\Sigma$. The data used in the definition of the quasi-local energy is the triple $\left(\sigma,|H|, \alpha_{H}\right)$ on $\Sigma$ where $\sigma$ is the induced metric, $|H|$ is the norm of the mean curvature vector, and $\alpha_{H}$ is the connection 1-form of the normal bundle with respect to the mean curvature vector

$$
\alpha_{H}(\cdot)=\left\langle\nabla_{(\cdot)}^{N} \frac{J}{|H|}, \frac{H}{|H|}\right\rangle
$$

where $\nabla^{N}$ is the covariant derivative in $N$.

Given an isometric embedding $Y$ of $\Sigma$ into the AdS space and the observer Killing field $\frac{\partial}{\partial t}$, let $\tau$ be the restriction of $t$ to $Y(\Sigma)$. Suppose the projection $\widehat{Y}$ of $Y(\Sigma)$ onto the static slice $t=0$ is embedded, and denote the induced metric, the second fundamental form, and the mean curvature of the image surface $\widehat{\Sigma}$ of $\widehat{Y}$ by $\hat{\sigma}_{a b}, \hat{h}_{a b}$, and $\widehat{H}$, respectively. The quasi-local energy $E\left(\Sigma, Y, \frac{\partial}{\partial t}\right)$ of $\Sigma$ with respect to the pair $\left(Y, \frac{\partial}{\partial t}\right)$ is

$$
\begin{aligned}
E\left(\Sigma, Y, \frac{\partial}{\partial t}\right)=\frac{1}{8 \pi}\{ & \int V \widehat{H} d \widehat{\Sigma}-\int\left[\sqrt{\left(1+V^{2}|\nabla \tau|^{2}\right)|H|^{2} V^{2}+\operatorname{div}\left(V^{2} \nabla \tau\right)^{2}}\right. \\
& \left.\left.-\operatorname{div}\left(V^{2} \nabla \tau\right) \sinh ^{-1} \frac{\operatorname{div}\left(V^{2} \nabla \tau\right)}{V|H| \sqrt{1+V^{2}|\nabla \tau|^{2}}}-V^{2} \alpha_{H}(\nabla \tau)\right] d \Sigma\right\},
\end{aligned}
$$

where $\nabla$ and div are the covariant derivatives and the divergence with respect to the induced metric $\sigma$ of the surface $\Sigma$, respectively.

Let $H_{0}$ and $\alpha_{H_{0}}$ be the mean curvature vector and the connection form of $Y(\Sigma)$ in the AdS space. In terms of $H_{0}$ and $\alpha_{H_{0}}$, we have

$$
\begin{aligned}
E\left(\Sigma, Y, \frac{\partial}{\partial t}\right)=\frac{1}{8 \pi}\left\{\int\right. & {\left[\sqrt{\left(1+V^{2}|\nabla \tau|^{2}\right)\left|H_{0}\right|^{2} V^{2}+\operatorname{div}\left(V^{2} \nabla \tau\right)^{2}}\right.} \\
& \left.-\operatorname{div}\left(V^{2} \nabla \tau\right) \sinh ^{-1} \frac{\operatorname{div}\left(V^{2} \nabla \tau\right)}{V\left|H_{0}\right| \sqrt{1+V^{2}|\nabla \tau|^{2}}}-V^{2} \alpha_{H_{0}}(\nabla \tau)\right] d \Sigma \\
-\int & {\left[\sqrt{\left(1+V^{2}|\nabla \tau|^{2}\right)|H|^{2} V^{2}+\operatorname{div}\left(V^{2} \nabla \tau\right)^{2}}\right.} \\
& \left.\left.-\operatorname{div}\left(V^{2} \nabla \tau\right) \sinh ^{-1} \frac{\operatorname{div}\left(V^{2} \nabla \tau\right)}{V|H| \sqrt{1+V^{2}|\nabla \tau|^{2}}}-V^{2} \alpha_{H}(\nabla \tau)\right] d \Sigma\right\} .
\end{aligned}
$$

While the above expressions seems to depend on the choice of the static chart, we can rewrite it purely in terms of the isometric embedding $Y$ and the observer $\frac{\partial}{\partial t}$. In fact,

$$
\begin{aligned}
V^{2} & =-\left\langle\frac{\partial}{\partial t}, \frac{\partial}{\partial t}\right\rangle \\
V^{2} \nabla \tau & =-\left(\frac{\partial}{\partial t}\right)^{\top},
\end{aligned}
$$


where $\left(\frac{\partial}{\partial t}\right)^{\top}$ denotes the tangential component of $\frac{\partial}{\partial t}$ to $Y(\Sigma)$. This allows us to define $E\left(\Sigma, Y, T_{0}\right)$ for each pair of an isometric embedding $Y$ and an observer Killing field $T_{0}$ using (3.1) via (3.3). Equivalently, we can define $E\left(\Sigma, Y, T_{0}\right)$ as follows:

Definition 1. The quasi-local energy $E\left(\Sigma, Y, T_{0}\right)$ of $\Sigma$ with respect to the pair $\left(Y, T_{0}\right)$ of an isometric embedding $Y$ and an observer $T_{0}$ is

$$
\begin{aligned}
& 8 \pi E\left(\Sigma, Y, T_{0}\right) \\
= & \int_{\Sigma}\left[\sqrt{-\left\langle T_{0}^{\perp}, T_{0}^{\perp}\right\rangle\left|H_{0}\right|^{2}+\operatorname{div}\left(T_{0}^{\top}\right)^{2}}-\operatorname{div}\left(T_{0}^{\top}\right) \sinh ^{-1} \frac{\operatorname{div}\left(T_{0}^{\top}\right)}{\left|H_{0}\right| \sqrt{-\left\langle T_{0}^{\perp}, T_{0}^{\perp}\right\rangle}}+\alpha_{H_{0}}\left(T_{0}^{\top}\right)\right] d \Sigma \\
& -\int_{\Sigma}\left[\sqrt{-\left\langle T_{0}^{\perp}, T_{0}^{\perp}\right\rangle|H|^{2}+\operatorname{div}\left(T_{0}^{\top}\right)^{2}}-\operatorname{div}\left(T_{0}^{\top}\right) \sinh ^{-1} \frac{\operatorname{div}\left(T_{0}^{\top}\right)}{|H| \sqrt{-\left\langle T_{0}^{\perp}, T_{0}^{\perp}\right\rangle}}+\alpha_{H}\left(T_{0}^{\top}\right)\right] d \Sigma .
\end{aligned}
$$

where $T_{0}^{\perp}$ is the normal part of $T_{0}$ to $Y(\Sigma)$.

Remark 3. From the above formulation, it follows that the quasi-local energy $E\left(\Sigma, Y, T_{0}\right)$ is equivariant. Namely, that the energy is invariant if an isometry of the AdS space acts on $Y$ and $T_{0}$ at the same time.

It is convenient to rewrite the quasi-local energy in terms of the quasi-local energy density and the quasi-local momentum density.

Definition 2. The quasi-local energy density with respect to $\left(Y, T_{0}\right)$ is defined to be

$$
f=\frac{\sqrt{\left|H_{0}\right|^{2}+\frac{\operatorname{div}\left(V^{2} \nabla \tau\right)^{2}}{V^{2}+V^{4}|\nabla \tau|^{2}}}-\sqrt{|H|^{2}+\frac{\operatorname{div}\left(V^{2} \nabla \tau\right)^{2}}{V^{2}+V^{4}|\nabla \tau|^{2}}}}{V \sqrt{1+V^{2}|\nabla \tau|^{2}}} .
$$

The quasi-local momentum density with respect to $\left(Y, T_{0}\right)$ is defined to be

$$
j=f V^{2} d \tau-d\left[\sinh ^{-1}\left(\frac{f d i v\left(V^{2} \nabla \tau\right)}{\left|H_{0}\right||H|}\right)\right]-\alpha_{H_{0}}+\alpha_{H} .
$$

In terms of $f$ and $j$, we have

$$
E\left(\Sigma, Y, T_{0}\right)=-\frac{1}{8 \pi} \int_{\Sigma}\left[\left\langle T_{0}, T_{0}\right\rangle f+j\left(T_{0}^{\top}\right)\right] d \Sigma
$$

The first variation of the quasi-local energy is evaluated in [13, Theorem 5.4]. It will be used later in Lemma 6.2. For reader's convenience, the formula will be recalled in the proof of Lemma 6.2.

\section{The SMall SPheres}

We setup the small sphere limit as in [12]. Let $p$ be a point in a spacetime $N$. Let $C_{p}$ be the future null hypersurface generated by future null geodesics starting at $p$. Pick any future directed timelike unit vector $e_{0}$ at $p$. Using $e_{0}$, we normalize a null vector $L$ at $p$ by

$$
\left\langle L, e_{0}\right\rangle=-1 \text {. }
$$


We consider the null geodesics of the normalized $L$ and let $r$ be the affine parameter of these null geodesics. Let $\Sigma_{r}$ be the family of surfaces on $C_{p}$ defined by the level sets of the affine parameter $r$. The inward null normal $\underline{L}$ of $\Sigma_{r}$ is normalized so that

$$
\langle L, \underline{L}\rangle=-1 \text {. }
$$

We parametrize $\Sigma_{r}$ in the following way. Consider a smooth map

$$
X: S^{2} \times[0, \epsilon) \rightarrow N
$$

such that for each fixed point in $S^{2}, X(\cdot, r), r \in[0, \epsilon)$ is a null geodesic parametrized by the affine parameter $r$, with $X(\cdot, 0)=p$ and $\frac{\partial X}{\partial r}(\cdot, 0) \in T_{p} N$ a null vector such that $\left\langle\frac{\partial X}{\partial r}(\cdot, 0), e_{0}\right\rangle=-1$. Let $L=\frac{\partial X}{\partial r}$ be the null generator, $\nabla_{L}^{N} L=0$. We also choose a local coordinate system $\left\{u^{a}\right\}_{a=1,2}$ on $S^{2}$ such that $\partial_{a}=\frac{\partial X}{\partial u^{a}}, a=1,2$ form a tangent basis to $\Sigma_{r}$. Let $\underline{L}$ be the null normal vector field along $\Sigma_{r}$ such that $\langle L, \underline{L}\rangle=-1$. Denote

$$
\begin{aligned}
l_{a b} & =\left\langle\nabla_{\partial_{a}}^{N} \partial_{b}, L\right\rangle \\
n_{a b} & =\left\langle\nabla_{\partial_{a}}^{N} \partial_{b}, \underline{L}\right\rangle \\
\eta_{a} & =\left\langle\nabla_{L}^{N} \partial_{a}, \underline{L}\right\rangle
\end{aligned}
$$

for the second fundamental forms in the direction of $L$ and $\underline{L}$ and the connection 1-form in the null normal frame, respectively. We consider these as tensors on $S^{2}$ depending on $r$ and use the induced metric on $\Sigma_{r}, \sigma_{a b}=\left\langle\partial_{a}, \partial_{b}\right\rangle$, to raise or lower indexes. We have

$$
\begin{aligned}
\nabla_{\partial_{a}}^{N} L & =-l_{a}^{c} \partial_{c}-\eta_{a} L \\
\nabla_{\partial_{a}}^{N} \partial_{b} & =\gamma_{a b}^{c} \partial_{c}-l_{a b} \underline{L}-n_{a b} L \\
\nabla_{\partial_{a}}^{N} \underline{L} & =-n_{a}^{c} \partial_{c}+\eta_{a} \underline{L},
\end{aligned}
$$

where $\gamma_{a b}^{c}$ are the Christoffel symbols of $\sigma_{a b}$. Let

$$
\begin{aligned}
& \hat{l}_{a b}=l_{a b}-\frac{1}{2}\left(\sigma^{c d} l_{c d}\right) \sigma_{a b} \\
& \hat{n}_{a b}=l_{a b}-\frac{1}{2}\left(\sigma^{c d} l_{c d}\right) \sigma_{a b}
\end{aligned}
$$

be the traceless part of $l_{a b}$ and $n_{a b}$.

The following identities for covariant derivatives are useful.

$$
\begin{aligned}
\nabla_{L}^{N} \partial_{a} & =-l_{a}^{c} \partial_{c}-\eta_{a} L \\
\nabla_{L}^{N} \underline{L} & =-\eta^{b} \partial_{b} .
\end{aligned}
$$

We consider $\sigma_{a b}, l_{a b}, n_{a b}, \eta_{a}$ as tensors on $S^{2} \times[0, \epsilon)$, or tensors on $S^{2}$ that depend on the parameter $r$. We shall see below that they have the following expansions.

$$
\sigma_{a b}=\tilde{\sigma}_{a b} r^{2}+O\left(r^{3}\right), l_{a b}=-\tilde{\sigma}_{a b} r+O\left(r^{2}\right), n_{a b}=\frac{1}{2} \tilde{\sigma}_{a b} r+O\left(r^{2}\right), \eta_{a}=\frac{1}{3} \beta_{a} r^{2}+O\left(r^{3}\right)
$$


where $\beta_{a}=\lim _{r \rightarrow 0} R_{L a L \underline{L}}$ is considered as a $(0,1)$ tensor on $S^{2}, \tilde{\sigma}_{a b}$ denotes the standard metric on unit $S^{2}$. Let $\tilde{\nabla}$ and $\tilde{\Delta}$ be the covariant derivative and the Laplacian with respect to $\tilde{\sigma}_{a b}$, respectively.

We shall also consider the pull-back of tensors from the null hypersurface. For example, we consider $R(L, \cdot, L, \underline{L})$ as a tensor defined on $C_{p}$ and take its pull-back through (4.1), which is then consider as a $(0,1)$ tenors on $S^{2}$ that depends on $r$ (or on $S^{2} \times[0, \epsilon)$ ). We shall abuse the notations and still denote the pull-back tensor by $R_{L a L L}$. In particular, $R_{L a b L}, R_{L a L \underline{L}}, R_{L \underline{L L L}}$ are considered as $r$ dependent $(0,2)$ tensor, $(0,1)$ tensor, and a scalar function on $S^{2}$, respectively, of the following orders

$$
R_{L a b L}=O\left(r^{2}\right), R_{L a L \underline{L}}=O(r) \text { and } R_{L \underline{L L} \underline{L}}=O(1) .
$$

We first write down the expansions of $L$ and $\partial_{a}$. Let $x^{0}, x^{i}, i=1,2,3$ be a normal coordinates system at $p$ such that the original future timelike vector $e_{0} \in T_{p} N$ is $\frac{\partial}{\partial x^{0}}$. The parametrization (4.1) is given by

$$
X\left(u^{a}, r\right)=X^{0}\left(u^{a}, r\right) \frac{\partial}{\partial x^{0}}+X^{i}\left(u^{a}, r\right) \frac{\partial}{\partial x^{i}}
$$

with the following expansions:

$$
\begin{aligned}
& X^{0}\left(u^{a}, r\right)=r+O\left(r^{2}\right) \\
& X^{i}\left(u^{a}, r\right)=r \tilde{X}^{i}\left(u^{a}\right)+O\left(r^{2}\right),
\end{aligned}
$$

where $\tilde{X}^{i}\left(u^{a}\right)$ are the three first eigenfunctions of the standard metric $\tilde{\sigma}_{a b}$ on $S^{2}$. For example, if we take the coordinates $u_{a}, a=1,2$ to be the standard spherical coordinate system $\theta, \phi$ with $\tilde{\sigma}=d \theta^{2}+\sin ^{2} \theta d \phi^{2}$, then $\tilde{X}^{1}=\sin \theta \sin \phi, \tilde{X}^{2}=\sin \theta \cos \phi$, and $\tilde{X}^{3}=\cos \theta$. In particular,

$$
\begin{aligned}
L & =\frac{\partial X}{\partial r}=\frac{\partial}{\partial x^{0}}+\tilde{X}^{i}\left(u^{a}\right) \frac{\partial}{\partial x^{i}}+O(r) \\
\partial_{a} & =\frac{\partial X}{\partial u^{a}}=r \frac{\partial \tilde{X}^{i}}{\partial u^{a}} \frac{\partial}{\partial x^{i}}+O\left(r^{2}\right), a=1,2 .
\end{aligned}
$$

\section{The Expansion of the Physical Data}

In this section, we compute the expansions of the induced metric, the second fundamental forms and the connection 1-form of $\Sigma_{r}$. We compute the expansion of the geometric quantities in terms of the affine parameter $r$. In the first subsection, we state the expansion in non-vacuum spacetimes. The result is exactly the same as in Section 3.1 of [12]. We collect these results in the first subsection to be used later. In the second subsection, we derive the expansion in vacuum spacetimes with $\Lambda=-3$.

5.1. Leading order expansion in non-vacuum spacetimes. The geometric quantities satisfy the following differential equations: 
Lemma 5.1. The induced metric, the second fundamental forms and the connection 1-form satisfy the following differential equations:

$$
\begin{gathered}
\partial_{r} \sigma_{a b}=-2 l_{a b} \\
\partial_{r} l_{a b}=R_{L a b L}-l_{a c} l_{b}^{c} \\
\partial_{r} n_{a b}=R_{L a b \underline{L}}-l_{b}^{c} n_{a c}+\nabla_{a} \eta_{b}-\eta_{a} \eta_{b} \\
\partial_{r} \eta_{a}=R_{L a L \underline{L}}+l_{a}^{b} \eta_{b} \\
\partial_{r}\left(\sigma^{a b} l_{a b}\right)=\frac{1}{2}\left(\sigma^{a b} l_{a b}\right)^{2}+\hat{l}_{a}^{b} \hat{l}_{b}^{a}+\operatorname{Ric}(L, L) \\
\partial_{r}\left(\sigma^{a b} n_{a b}\right)=\operatorname{Ric}(L, \underline{L})+R_{L \underline{L L} \underline{L}}+l^{a b} n_{a b}+\operatorname{div} v_{\sigma} \eta-\eta_{a} \eta^{a} .
\end{gathered}
$$

Ric and $R_{\alpha \beta \gamma \delta}$ are the Ricci curvature and the full Riemannian curvature tensor of the spacetime $N$, respectively.

We have the following expansions for the curvature tensor:

$$
\begin{aligned}
R_{L a b L} & =r^{2} \bar{R}_{L a b L}+O\left(r^{3}\right) \\
R_{L a L \underline{L}} & =r \bar{R}_{L a L \underline{L}}+O\left(r^{2}\right) \\
R_{L \underline{L L} \underline{L}} & =\bar{R}_{L \underline{L L} \underline{L}}+O(r),
\end{aligned}
$$

where $\bar{R}_{L a b L}, \bar{R}_{L a L \underline{L}}$ and $\bar{R}_{L \underline{L L} \underline{L}}$ correspond to the appropriate rescaled limit of the respective tensors as $r \rightarrow 0$. For example,

$$
\bar{R}_{L a b L}=\lim _{r \rightarrow 0} \frac{1}{r^{2}} R_{L a b L}=R\left(\frac{\partial}{\partial x^{0}}+\tilde{X}^{i} \frac{\partial}{\partial x^{i}}, \frac{\partial \tilde{X}^{j}}{\partial u^{a}}, \frac{\partial \tilde{X}^{k}}{\partial u^{b}}, \frac{\partial}{\partial x^{0}}+\tilde{X}^{l} \frac{\partial}{\partial x^{l}}\right)(p) .
$$

It is considered as a $(0,2)$ tensor on the standard $S^{2}$.

Lemma 5.2. We have the following expansions:

$$
\begin{aligned}
l_{a b} & =-r \tilde{\sigma}_{a b}+\frac{2}{3} r^{3} \bar{R}_{L a b L}+O\left(r^{4}\right) \\
\sigma_{a b} & =r^{2} \tilde{\sigma}_{a b}-\frac{1}{3} r^{4} \bar{R}_{L a b L}+O\left(r^{5}\right) \\
l_{a}^{c} & =-r^{-1} \delta_{a}^{c}+\frac{1}{3} r \bar{R}_{L a b L} \tilde{\sigma}^{b c}+O\left(r^{2}\right) \\
\eta_{a} & =\frac{1}{3} r^{2} \bar{R}_{L a L \underline{L}}+O\left(r^{3}\right) \\
\sigma^{a b} l_{a b} & =-\frac{2}{r}+\frac{1}{3} r \bar{R} i c(L, L)+O\left(r^{2}\right) \\
\sigma^{a b} n_{a b} & =\frac{1}{r}+r\left[\bar{R}_{L} \underline{L} L \underline{L}+\frac{2}{3} \bar{R} i c(L, \underline{L})+\frac{1}{6} \bar{R} i c(L, L)\right]+O\left(r^{2}\right) .
\end{aligned}
$$

In summary, we have the following expansions on the surfaces $\Sigma_{r}$ : 
Lemma 5.3. We have the following expansions for the data $\left(\sigma,|H|, \operatorname{div} \alpha_{H}\right)$ on $S^{2}$ :

$$
\begin{aligned}
\sigma_{a b}= & r^{2} \tilde{\sigma}_{a b}-\frac{1}{3} r^{4} \bar{R}_{L a b L}+O\left(r^{5}\right) \\
|H|^{2}= & \frac{4}{r^{2}}+\left[2 \bar{R}_{L \underline{L} L \underline{L}}+\frac{4}{3} \bar{R} i c(L, \underline{L})+\frac{1}{3} \bar{R} i c(L, L)\right]+O(r) \\
\operatorname{div}_{\sigma} \alpha_{H}= & \tilde{\Delta}\left[\frac{1}{2} \bar{R}_{L \underline{L} L \underline{L}}+\frac{1}{6} \bar{R} i c(L, L)+\frac{1}{3} \bar{R} i c(L, \underline{L})\right] \\
& -\bar{R}_{L \underline{L} L \underline{L}}-\frac{1}{3} \bar{R} i c(L, \underline{L})-\frac{1}{6} \bar{R} i c(L, L)+O(r) .
\end{aligned}
$$

5.2. Further expansions in vacuum spacetimes. In this subsection, we assume the spacetime is vacuum and compute the higher order terms in the expansions for the physical data. Enough expansions are obtained to evaluate the leading term of the small sphere limit of the quasi-local energy. In a vacuum spacetime, the only non-trivial components of the curvature tensor are the Weyl curvature tensor. We have

$$
R_{\alpha \beta \gamma \delta}=W_{\alpha \beta \gamma \delta}+\kappa^{2}\left(g_{\alpha \gamma} g_{\beta \delta}-g_{\alpha \delta} g_{\beta \gamma}\right)
$$

and

$$
R i c_{\alpha \delta}=-3 \kappa^{2} g_{\alpha \delta}
$$

In terms of the null frame $\left\{e_{a}, L, \underline{L}\right\}$, we have

$$
\begin{aligned}
R_{a L b L} & =W_{a L b L} \\
R_{a L b \underline{L}} & =W_{a \underline{L} \underline{L}}-\kappa^{2} g_{a b} \\
R_{a L L \underline{L}} & =W_{a L L \underline{L}} \\
R_{L \underline{L L} \underline{L}} & =W_{L \underline{L L} \underline{L}}-\kappa^{2} .
\end{aligned}
$$

We decompose the Weyl curvature tensor at the point $p$ using the null frame $\left\{e_{a}, L, \underline{L}\right\}$ following the notation of Christodoulou and Klainerman in [14]:

$$
\begin{aligned}
\alpha_{a b} & =\bar{W}_{a L b L} & \underline{\alpha}_{a b} & =\bar{W}_{a \underline{L} b \underline{L}} \\
\beta_{a} & =\bar{W}_{a L L L} & \underline{\beta}_{a} & =\bar{W}_{a \underline{L L} L} \\
\rho & =\bar{W}_{\underline{L} L \underline{L} L} & \sigma & =\epsilon^{a b} \bar{W}_{a b \underline{L} L} .
\end{aligned}
$$

From the vacuum condition and the Bianchi equations, we obtain the following relations:

$$
\begin{aligned}
\bar{W}_{L a b \underline{L}} & =\frac{1}{2} \tilde{\sigma}_{a b} \rho+\frac{1}{4} \epsilon_{a b} \sigma \\
\bar{W}_{a b c L} & =-\epsilon_{a b} \epsilon_{c d} \beta^{d} \\
\bar{W}_{a b c \underline{L}} & =\epsilon_{a b} \epsilon_{c d} \underline{\beta}^{d} \\
\bar{W}_{a b \underline{L} L} & =\frac{1}{2} \epsilon_{a b} \sigma .
\end{aligned}
$$


All $\alpha, \underline{\alpha}, \beta, \beta, \rho$ and $\sigma$ are considered as tensors on $S^{2}$ through the limiting process described above. In particular, the covariant derivatives of them with respect to the standard metric $\tilde{\sigma}_{a b}$ can be computed as follows.

Lemma 5.4. [12, Lemma 3.6]

$$
\begin{aligned}
\tilde{\nabla}_{c} \alpha_{a b} & =\left(\tilde{\sigma}_{c a} \tilde{\sigma}_{b d}+\tilde{\sigma}_{c b} \tilde{\sigma}_{a d}+\epsilon_{c a} \epsilon_{b d}+\epsilon_{c b} \epsilon_{a d}\right) \beta^{d} \\
\tilde{\nabla}_{c} \underline{\alpha}_{a b} & =\frac{1}{2}\left(\tilde{\sigma}_{c a} \tilde{\sigma}_{b d}+\tilde{\sigma}_{c b} \tilde{\sigma}_{a d}+\epsilon_{c a} \epsilon_{b d}+\epsilon_{c b} \epsilon_{a d}\right) \underline{\beta}^{d} \\
\tilde{\nabla}_{a} \beta_{b} & =-\frac{3}{4} \sigma \epsilon_{a b}+\frac{3}{2} \rho \tilde{\sigma}_{a b}-\frac{1}{2} \alpha_{a b} \\
\tilde{\nabla}_{a} \underline{\beta}_{b} & =\frac{3}{8} \sigma \epsilon_{a b}+\frac{3}{4} \rho \tilde{\sigma}_{a b}-\underline{\alpha}_{a b} \\
\tilde{\nabla}_{a} \rho & =-\beta_{a}-2 \underline{\beta}_{a} \\
\tilde{\nabla}_{a} \sigma & =2 \epsilon_{a b}\left(\beta^{b}-2 \underline{\beta}^{b}\right) .
\end{aligned}
$$

Contracting with respect to $\tilde{\sigma}_{a b}$ and $\epsilon_{a b}$, we obtain the following formulae:

Lemma 5.5. [12, Lemma 3.7]

$$
\begin{aligned}
\tilde{\nabla}^{a} \alpha_{a b} & =4 \beta_{b}, \epsilon^{c a} \nabla_{c} \alpha_{a b}=4 \epsilon_{b d} \beta^{d} \\
\tilde{\nabla}^{a} \underline{\alpha}_{a b} & =2 \underline{\beta}_{b}, \epsilon^{c a} \nabla_{c} \underline{\alpha}_{a b}=2 \epsilon_{b d} \underline{\beta}^{d} \\
\tilde{\nabla}^{a} \beta_{a} & =3 \rho, \epsilon^{a b} \tilde{\nabla}_{a} \beta_{b}=-\frac{3}{2} \sigma \\
\tilde{\nabla}^{a} \underline{\beta}_{a} & =\frac{3}{2} \rho, \epsilon^{a b} \tilde{\nabla}_{a} \underline{\beta}_{b}=\frac{3}{4} \sigma .
\end{aligned}
$$

In particular, it follows that $\tilde{\Delta} \rho=-6 \rho$ and $\tilde{\Delta} \sigma=-6 \sigma$.

The covariant derivative in the spacetime $N$ at $p$ in the direction of $L$ is denoted by the symbol $D$. For example,

$$
D \alpha_{a b}=\nabla_{L}^{N} W\left(e_{a}, L, e_{b}, L\right)(p)
$$

$D \alpha_{a b}$ is also considered as a tensor on $S^{2}$ through the limiting process and its covariant derivatives with respect to the standard metric $\tilde{\sigma}_{a b}$ can be computed in the same manner. Relations similar to equation (5.17) hold among $D$ of the Weyl curvature components.

Lemma 5.6. [12, Lemma 3.9]

$$
\begin{aligned}
\tilde{\nabla}^{a} D \beta_{a} & =4 D \rho \\
\tilde{\nabla}^{a} D^{2} \beta_{a} & =5 D^{2} \rho \\
\tilde{\nabla}^{a}\left(D \alpha_{a b}\right) & =5 D \beta_{b} \\
\tilde{\nabla}^{a}\left(D^{2} \alpha_{a b}\right) & =6 D^{2} \beta_{b}
\end{aligned}
$$

We have the following expansions for the Weyl curvature tensor. 
Lemma 5.7. [12, Lemma 3.10]

$$
\begin{aligned}
& W_{L a L \underline{L}}=r \beta_{a}+r^{2} D \beta_{a}+\frac{1}{2} r^{3} D^{2} \beta_{a}+O\left(r^{4}\right) \\
& W_{L \underline{L} L \underline{L}}=\rho+r D \rho+r^{2}\left[\frac{1}{2} D^{2} \rho-\frac{1}{3}|\beta|^{2}\right]+O\left(r^{3}\right) .
\end{aligned}
$$

We are now ready to compute the expansion of the physical data:

Lemma 5.8. We have the following expansions for $\sigma^{a b} l_{a b}, \sigma^{a b} n_{a b}$ and $\eta_{a}$.

and

where

$$
\begin{gathered}
\sigma^{a b} l_{a b}=-\frac{2}{r}+\frac{1}{45} r^{3}|\alpha|^{2}+O\left(r^{4}\right) \\
\sigma^{a b} n_{a b}=\frac{1}{r}+r\left(\sigma^{a b} n_{a b}\right)^{(1)}+r^{2}\left(\sigma^{a b} n_{a b}\right)^{(2)}+r^{3}\left(\sigma^{a b} n_{a b}\right)^{(3)}+O\left(r^{4}\right)
\end{gathered}
$$

$$
\eta_{a}=\frac{r^{2}}{3} \beta_{a}+\frac{r^{3}}{4} D \beta_{a}+r^{4}\left[\frac{1}{10} D^{2} \beta_{a}-\frac{1}{45} \alpha_{a b} \beta^{b}\right]+O\left(r^{5}\right)
$$

Proof. We rewrite $l_{a b}$ as

$$
\begin{aligned}
& \left(\sigma^{a b} n_{a b}\right)^{(1)}=\rho+\kappa^{2} \\
& \left(\sigma^{a b} n_{a b}\right)^{(2)}=\frac{2}{3} D \rho \\
& \left(\sigma^{a b} n_{a b}\right)^{(3)}=\frac{3}{8} D^{2} \rho+\frac{1}{30}|\alpha|^{2}-\frac{11}{45}|\beta|^{2} .
\end{aligned}
$$

$$
\begin{aligned}
l_{a b} & =-r \tilde{\sigma}_{a b}-\frac{2}{3} r^{3} \alpha_{a b}+O\left(r^{4}\right) \\
& =\left(-r \tilde{\sigma}_{a b}-\frac{1}{3} r^{3} \alpha_{a b}\right)-\frac{1}{3} r^{3} \alpha_{a b}+O\left(r^{4}\right) .
\end{aligned}
$$

Hence, $\hat{l}_{a b}$, the traceless part of $l_{a b}$, is given by

$$
\hat{l}_{a b}=-\frac{1}{3} r^{3} \alpha_{a b}+O\left(r^{4}\right) .
$$

It follows that

$$
\sigma^{a b} l_{a b}=-\frac{2}{r}+\frac{r^{3}}{45}|\alpha|^{2}+O\left(r^{4}\right)
$$

Next we compute $\eta_{a}$. Let

$$
\eta_{a}=r^{2} \eta_{a}^{(2)}+r^{3} \eta_{a}^{(3)}+r^{4} \eta_{a}^{(4)}+O\left(r^{5}\right)
$$

From Lemma 5.2, we have

Equation (5.4) is equivalent to

$$
\eta_{a}=\frac{1}{3} r^{2} \beta_{a}+O\left(r^{3}\right) .
$$

$$
r^{-1} \partial_{r}\left(r \eta_{a}\right)=W_{L a L \underline{L}}+\left(l_{a}^{b}+r^{-1} \delta_{a}^{b}\right) \eta_{b} .
$$


By equation (5.19), the right hand side can be expanded into

$$
r \beta_{a}+r^{2} D \beta_{a}+r^{3}\left[\frac{1}{2} D^{2} \beta_{a}-\frac{1}{9} \alpha_{a b} \beta^{b}\right]+O\left(r^{4}\right) .
$$

Integrating, we obtain

$$
\begin{aligned}
\eta_{a}^{(3)} & =\frac{1}{4} D \beta_{a} \\
\eta_{a}^{(4)} & =\frac{1}{10} D^{2} \beta_{a}-\frac{1}{45} \alpha_{a b} \beta^{b} .
\end{aligned}
$$

For $n_{a b}$, we start with equation (5.3). It is equivalent to

$$
\begin{aligned}
r \partial_{r}\left(r^{-1} n_{a b}\right) & =R_{L a b \underline{L}}-\left(l_{b}^{c}+r^{-1} \delta_{b}^{c}\right) n_{a c}+\nabla_{a} \eta_{b}-\eta_{a} \eta_{b} \\
& =W_{L a b \underline{L}}+\sigma_{a b}-\left(l_{b}^{c}+r^{-1} \delta_{b}^{c}\right) n_{a c}+\nabla_{a} \eta_{b}-\eta_{a} \eta_{b} .
\end{aligned}
$$

The equation becomes

$$
\begin{aligned}
r \partial_{r}\left(r^{-1} n_{a b}\right) & =r^{2}\left[\bar{W}_{L a b \underline{L}}+\kappa^{2} \tilde{\sigma}_{a b}-\frac{1}{6} \bar{W}_{L a b L}+\tilde{\nabla}_{a} \eta_{b}^{(2)}\right]+O\left(r^{3}\right) \\
& =r^{2}\left(\rho+\kappa^{2}\right) \tilde{\sigma}_{a b}+O\left(r^{3}\right) .
\end{aligned}
$$

Integrating, we obtain

$$
n_{a b}=\frac{1}{2} r \tilde{\sigma}_{a b}+\frac{1}{2} r^{3}\left(\rho+\kappa^{2}\right) \tilde{\sigma}_{a b}+O\left(r^{4}\right) .
$$

Lastly, we deal with equation (5.6) for $\sigma^{a b} n_{a b}$. We decompose

$$
\begin{aligned}
l^{a b} n_{a b} & =l_{a b} \sigma^{a c} \sigma^{b d} n_{c d} \\
& =\left(l_{a b}+\frac{\sigma_{a b}}{r}\right) \sigma^{a c} \sigma^{b d}\left(n_{c d}-\frac{\sigma_{c d}}{2 r}\right)+\frac{1}{2} r^{-1} \sigma^{a b} l_{a b}-r^{-1} \sigma^{a b} n_{a b}+r^{-2} .
\end{aligned}
$$

Thus equation (5.6) is equivalent to

$r^{-1} \partial_{r}\left(r \sigma^{a b} n_{a b}\right)=r^{-2}+\frac{1}{2} r^{-1} \sigma^{a b} l_{a b}+\left(l^{a b}+r^{-1} \sigma^{a b}\right)\left(n_{a b}-\frac{1}{2} r^{-1} \sigma_{a b}\right)-\eta_{a} \eta^{a}+W_{L \underline{L} L \underline{L}}+2 \kappa^{2}+d i v_{\sigma} \eta$.

Notice that

$$
\begin{aligned}
l_{a b}+r^{-a} \sigma_{a b} & =-\frac{1}{3} r^{3} \alpha_{a b}+O\left(r^{4}\right) \\
n_{c d}-\frac{1}{2} r^{-1} \sigma_{c d} & =r^{3}\left(\frac{1}{2}\left(\rho+\kappa^{2}\right) \tilde{\sigma}_{a b}-\frac{1}{6} \alpha_{a b}\right)+O\left(r^{4}\right) .
\end{aligned}
$$

We have

$$
r^{-1} \partial_{r}\left(r \sigma^{a b} n_{a b}\right)=r^{2}\left[\frac{1}{15}|\alpha|^{2}-\frac{1}{9}|\beta|^{2}\right]+d i v_{\sigma} \eta+W_{L \underline{L} L \underline{L}}+2 \kappa^{2}+O\left(r^{3}\right) .
$$

Integrating this equation, we obtain the expansion for $\sigma^{a b} n_{a b}$. 


$$
\begin{aligned}
\sigma^{a b} n_{a b}= & \frac{1}{r}+\frac{r}{2}\left[\left(\rho+2 \kappa^{2}+\left(\operatorname{div}_{\sigma} \eta\right)^{(0)}\right]+\frac{r^{2}}{3}\left[D \rho+\left(\operatorname{div}_{\sigma} \eta\right)^{(1)}\right]+\frac{r^{3}}{4}\left[\frac{1}{2}\left(D^{2} \rho-\frac{2}{3}|\beta|^{2}\right)\right.\right. \\
& \left.+\left(\operatorname{div}_{\sigma} \eta\right)^{(2)}-\frac{1}{9}|\beta|^{2}+\frac{1}{15}|\alpha|^{2}\right]+O\left(r^{4}\right) .
\end{aligned}
$$

We compute

$$
\begin{aligned}
\left(\operatorname{div}_{\sigma} \eta\right)^{(0)} & =\frac{1}{3} \tilde{\nabla}^{a}\left(\beta_{a}\right)=\rho \\
\left(\operatorname{div}_{\sigma} \eta\right)^{(1)} & =\frac{1}{4} \tilde{\nabla}^{a} D \beta_{a}=D \rho \\
\left(\operatorname{div}_{\sigma} \eta\right)^{(2)} & =\frac{1}{10} \tilde{\nabla}^{a}\left(D^{2} \beta_{a}\right)-\frac{2}{15} \tilde{\nabla}^{a}\left(\alpha_{a b} \beta^{b}\right) \\
& =\frac{1}{2} D^{2} \rho+\frac{1}{15}\left(|\alpha|^{2}-8|\beta|^{2}\right) .
\end{aligned}
$$

which follows from the expansion of $\eta$ and the following expansion for $\gamma_{a b}^{c}$ :

$$
\gamma_{a b}^{c}=\tilde{\gamma}_{a b}^{c}+r^{2} \gamma_{a b}^{(2) c}+O\left(r^{3}\right)
$$

where $\tilde{\gamma}_{a b}^{c}$ is the Christoffel symbols for $\tilde{\sigma}_{a b}$ and $\gamma_{a b}^{(2) c}=-\frac{1}{6} \tilde{\sigma}^{c d}\left(\tilde{\nabla}_{a} \alpha_{d b}+\tilde{\nabla}_{b} \alpha_{a d}-\tilde{\nabla}_{d} \alpha_{a b}\right)$.

When we compute the small sphere limit in vacuum spacetimes, there are several functions and tensors on $S^{2}$ which appear repeatedly. These quantities are computed in [12]. We recall the results here. We define the functions $W_{0}, W_{i}$ and $P_{k}$ as follows:

$$
\begin{aligned}
& W_{0}=\tilde{X}^{i} \tilde{X}^{j} \bar{W}_{0 i 0 j}=\rho \\
& W_{i}=\tilde{X}^{j} \tilde{X}^{k} \bar{W}_{0 k i j}=\frac{1}{2}\left(\beta^{b}-2 \underline{\beta}^{b}\right) \tilde{\nabla}_{b} \tilde{X}^{i} \\
& P_{k}=\frac{1}{15} \bar{W}_{0 i 0 k} \tilde{X}^{i}-\frac{1}{6} W_{0} \tilde{X}^{k}=-\frac{1}{30}\left(\beta^{a}+2 \underline{\beta}^{a}\right) \tilde{\nabla}_{a} \tilde{X}^{k}-\frac{1}{10} \rho \tilde{X}^{k}
\end{aligned}
$$

$W_{i}$ are -6-eigenfunctions and $P_{k}$ are -12-eigenfunctions of the standard Laplacian on $S^{2}$.

Next, we introduce $R_{i j}$ and $S_{j}$. From the expansion of the induced metric $\sigma_{a b}$, we derive

$$
\sigma^{(0) a b}=-\frac{1}{3} \alpha^{a b} \text { and } \tilde{\sigma}^{a b} \gamma_{a b}^{(2) c}=-\frac{4}{3} \beta^{c} .
$$

$R_{i j}$ and $S_{j}$ are defined as follows:

$$
\begin{aligned}
R_{i j}= & \sigma^{(0) a b} \tilde{X}_{a}^{i} \tilde{X}_{b}^{j} \\
= & \frac{1}{3}\left[2 \tilde{X}^{i} \tilde{X}^{k} \bar{W}_{0 i 0 k}+2 \tilde{X}^{j} \tilde{X}^{k} \bar{W}_{0 k 0 i}+\tilde{X}^{i} \tilde{X}^{j} \tilde{X}^{n}\left(\bar{W}_{0 i n j}+\bar{W}_{0 j n i}\right)\right. \\
& \left.-2 \bar{W}_{0 i 0 j}-\rho \delta_{i j}-\rho \tilde{X}^{i} \tilde{X}^{j}-\tilde{X}^{n}\left(\bar{W}_{0 i n j}+\bar{W}_{0 j n i}\right)\right] \\
S_{j}= & \tilde{\sigma}^{a b} \gamma_{a b}^{(2) c} \tilde{X}_{c}^{j} \\
= & \frac{1}{3}\left(-4 \bar{W}_{0 j 0 n} \tilde{X}^{n}+4 \tilde{X}^{j} W_{0}+4 W_{j}\right) .
\end{aligned}
$$




\section{Optimal EmbedDing EQUation}

For the rest of the paper, we set $\kappa=1$. This corresponds to choosing $\Lambda=-3$ in the Einstein equation. In this section, we study the limiting behavior of the optimal embedding equation. We consider the Ads space to be embedded in $\mathbb{R}^{3,2}$ and consider isometric embeddings of the form:

$$
Y=\left(Y_{0}, Y_{i}, Y_{4}\right)
$$

where $Y_{0}, Y_{i}$ and $Y_{4}$ are the restriction of $y_{0}$ and $y_{i}$ and $y_{4}$ to the image of the isometric embedding.

Similar to the small sphere limit of the Wang-Yau quasi-local mass, we look for solutions of the optimal embedding equation $\left(Y, T_{0}\right)$ of the form

$$
\begin{aligned}
& Y_{0}=\sum_{i=3}^{\infty} Y_{0}^{(i)} r^{i} \\
& Y_{i}=r \tilde{X}^{i}+\sum_{k=3}^{\infty} Y_{i}^{(k)} r^{k} \\
& Y_{4}=\sqrt{1+\sum_{i} Y_{i}^{2}-Y_{0}^{2}}
\end{aligned}
$$

and

$$
T_{0}=A\left(y^{0} \frac{\partial}{\partial y^{4}}-y^{4} \frac{\partial}{\partial y^{0}}\right)-B_{i}\left(y^{0} \frac{\partial}{\partial y^{i}}+y^{i} \frac{\partial}{\partial y^{0}}\right)-C_{j}\left(y^{4} \frac{\partial}{\partial y^{j}}+y^{j} \frac{\partial}{\partial y^{4}}\right)+D_{p} \epsilon_{p q r} y^{q} \frac{\partial}{\partial y^{r}} .
$$

Remark 4. As mentioned in Remark 3, the quasi-local energy is equivariant and thus it is natural to consider the embedding of the above form and allow $T_{0}$ to be a general observer Killing field.

In this section, we compute the leading order term of the optimal embedding equation and use it to determine $Y_{i}^{(3)}$. While it is possible to also solve $Y_{0}^{(3)}$, the set of solutions is different for the vacuum case and the non-vacuum case. Hence, we defer the determination of $Y_{0}^{(3)}$ to Section 8 for vacuum spacetimes since it will only be needed for the limit in the vacuum case.

Recall that the isometric embedding equation of a metric $\sigma$ into the AdS space in $\mathbb{R}^{3,2}$ is

$$
\sum_{i} \partial_{a} Y_{i} \partial_{b} Y_{i}-\partial_{a} Y_{0} \partial_{b} Y_{0}-\partial_{a} Y_{4} \partial_{b} Y_{4}=\sigma_{a b}
$$

For the metric $\sigma_{a b}$ on $\Sigma_{r}$ given in (5.9) and the embedding $\left(Y_{0}, Y_{i}, Y_{4}\right)$ given in (6.1), the leading order term of the isometric embedding is the following system of linear equation on $Y_{i}^{(3)}$

$$
\partial_{a} \tilde{X}^{i} \partial_{b} Y_{i}^{(3)}+\partial_{b} \tilde{X}^{i} \partial_{a} Y_{i}^{(3)}=-\frac{1}{3} \bar{R}_{L a b L}=\frac{1}{3} \alpha_{a b}-\frac{1}{6} \bar{R} i c(L, L) \tilde{\sigma}_{a b}
$$




\section{Lemma 6.1.}

$$
Y_{i}^{(3)}=-\frac{1}{3} \beta^{c} \tilde{\nabla}_{c} \tilde{X}^{i}+\frac{1}{2} \rho \tilde{X}^{i}-\frac{1}{12} \bar{R} i c(L, L) \tilde{X}^{i}
$$

satisfies (6.2).

Proof. From Lemma 6.1 of [13], we know that

$$
\sum_{i} \partial_{a} \tilde{X}^{i} \partial_{b}\left(-\frac{1}{3} \beta^{c} \tilde{\nabla}_{c} \tilde{X}^{i}+\frac{1}{2} \rho \tilde{X}^{i}\right)+\partial_{b} \tilde{X}^{i} \partial_{a}\left(-\frac{1}{3} \beta^{c} \tilde{\nabla}_{c} \tilde{X}^{i}+\frac{1}{2} \rho \tilde{X}^{i}\right)=\frac{1}{3} \alpha_{a b} .
$$

On the other hand, it is easy to see that

$$
\sum_{i} \partial_{a} \tilde{X}^{i} \partial_{b}\left(\bar{R} i c(L, L) \tilde{X}^{i}\right)+\partial_{b} \tilde{X}^{i} \partial_{a}\left(\bar{R} i c(L, L) \tilde{X}^{i}\right)=2 \bar{R} i c(L, L) \tilde{\sigma}_{a b}
$$

This finishes the proof of the lemma.

The data on $\Sigma_{r}$ admit the following expansion

$$
\begin{aligned}
\sigma_{a b} & =r^{2} \tilde{\sigma}_{a b}+r^{4} \tilde{\sigma}_{a b}^{(4)}+O\left(r^{5}\right) \\
|H| & =\frac{2}{r}+h^{(1)} r+O\left(r^{2}\right) \\
\left(\alpha_{H}\right)_{a} & =\left(\alpha_{H}^{(2)}\right)_{a} r^{2}+O\left(r^{3}\right) .
\end{aligned}
$$

Similarly. the data on the image of the isometric embedding admit the following expansion

$$
\begin{aligned}
\left|H_{0}\right| & =\frac{2}{r}+h_{0}^{(1)} r+O\left(r^{2}\right) \\
\left(\alpha_{H_{0}}\right)_{a} & =\left(\alpha_{H_{0}}^{(2)}\right)_{a} r^{2}+O\left(r^{3}\right) .
\end{aligned}
$$

It follows that the quasi-local energy density $f$ admits the following expansion:

$$
f=f^{(1)} r+O\left(r^{2}\right)
$$

where $f^{(1)}=\frac{h_{0}^{(1)}-h^{(1)}}{A}$. In the following lemma, we derive the leading order term of the Euler-Lagrange equation for the quasi-local energy.

Lemma 6.2. For the observer

$$
T_{0}=A\left(y^{0} \frac{\partial}{\partial y^{4}}-y^{4} \frac{\partial}{\partial y^{0}}\right)-B_{i}\left(y^{0} \frac{\partial}{\partial y^{i}}+y^{i} \frac{\partial}{\partial y^{0}}\right)-C_{j}\left(y^{4} \frac{\partial}{\partial y^{j}}+y^{j} \frac{\partial}{\partial y^{4}}\right)+D_{p} \epsilon_{p q r} y^{q} \frac{\partial}{\partial y^{r}}
$$

and an isometric embedding of the form (6.1), the leading order term of the Euler-Lagrange equation is the following equation on $\left(A, C^{i}\right)$ and $Y_{0}^{(3)}$

$$
\frac{1}{2} \tilde{\Delta}(\tilde{\Delta}+2) Y_{0}^{(3)}=\tilde{\nabla}^{a}\left(\alpha_{H}^{(2)}\right)_{a}+\tilde{\nabla}^{a}\left(f^{(1)} \tilde{\nabla}_{a} C_{i} \tilde{X}^{i}\right)+\frac{1}{2} \tilde{\Delta}\left(f^{(1)} C_{i} \tilde{X}^{i}\right) .
$$


Proof. From [13, Theorem 5.4 ], the first variation of the quasi-local energy (up to a factor of $8 \pi)$ is

$$
\begin{aligned}
& \int_{\Sigma}(\delta \tau) \operatorname{div}\left[V^{2} \nabla \sinh ^{-1} \frac{f d i v\left(V^{2} \nabla \tau\right)}{\left|H_{0}\right||H|}-f V^{4} \nabla \tau+V^{2}\left(\alpha_{H_{0}}-\alpha_{H}\right)\right] d \Sigma \\
& +\int_{\Sigma} \delta Y^{i} \bar{\nabla}_{i} V\left[f V\left(1+2 V^{2}|\nabla \tau|^{2}\right)-2 V \nabla \tau \nabla \sinh ^{-1} \frac{f d i v\left(V^{2} \nabla \tau\right)}{\left|H_{0}\right||H|}+\left(\alpha_{H}-\alpha_{H_{0}}\right)(2 V \nabla \tau)\right] d \Sigma
\end{aligned}
$$

where $\delta \tau$ and $\delta Y^{i}$ are coupled through the isometric embedding equation. Recall that

$$
\begin{aligned}
V^{2} & =-\left\langle T_{0}, T_{0}\right\rangle=\left(A^{2}-\sum_{i} C_{i}^{2}\right)+O\left(r^{2}\right) \\
V^{2} \nabla_{a} \tau & =-\left(T_{0}^{T}\right)_{a}=r C_{i} \tilde{\nabla}_{a} \tilde{X}^{i}+O\left(r^{2}\right) .
\end{aligned}
$$

As a result,

$$
\operatorname{div}\left[V^{2} \nabla \sinh ^{-1} \frac{f \operatorname{div}\left(V^{2} \nabla \tau\right)}{\left|H_{0}\right||H|}-f V^{4} \nabla \tau+V^{2}\left(\alpha_{H_{0}}-\alpha_{H}\right)\right]=O(1)
$$

while

$$
f V\left(1+2 V^{2}|\nabla \tau|^{2}\right)-2 V \nabla \tau \nabla \sinh ^{-1} \frac{f d i v\left(V^{2} \nabla \tau\right)}{\left|H_{0}\right||H|}+\left(\alpha_{H}-\alpha_{H_{0}}\right)(2 V \nabla \tau)=O(r) .
$$

Moreover, from the linearized isometric embedding equation, we conclude that

$$
\delta Y^{i}=O\left(r^{2}\right) .
$$

As a result, the second integral in (6.3) is of higher order in $r$ compared to the first integral and the leading order term of the Euler-Lagrange equation is simply that the $\mathrm{O}(1)$ term of

$$
\operatorname{div}\left[V^{2} \nabla \sinh ^{-1} \frac{f d i v\left(V^{2} \nabla \tau\right)}{\left|H_{0}\right||H|}-f V^{4} \nabla \tau+V^{2}\left(\alpha_{H_{0}}-\alpha_{H}\right)\right]
$$

is equal to 0. From the formula in the proof of Theorem 6.2 of [13], we get

$$
\operatorname{div} \alpha_{H_{0}}=\frac{1}{2} \tilde{\Delta}(\tilde{\Delta}+2) Y_{0}^{(3)}
$$

by treating the image of the isometric embedding as a small perturbation of the isometric embedding into the hyperbolic space. The lemma follows from collecting terms.

\section{Small Sphere Limit of the QUASI-LOCAL EnERGY IN SPACETIMES With Matters}

In this section, we compute the small sphere limit of the quasi-local energy and show that it recovers the matter field at $p$. More precisely, we show the following:

Theorem 7.1. Let $\Sigma_{r}$ be the family of spheres approaching $p$ constructed in Section 4 and $\mathcal{P}$ denote the set of $\left(Y, T_{0}\right)$ admitting a power series expansion given in equation (6.1). 
(1) For any pair $\left(Y, T_{0}\right)$ in $\mathcal{P}$, we have

$$
\lim _{r \rightarrow 0} r^{-3} E\left(\Sigma_{r}, Y_{r}, T_{0}\right)=\frac{4 \pi}{3} T\left(e_{0}, A e_{0}+C_{i} e_{i}\right),
$$

where $T(\cdot, \cdot)$ is the stress-energy tensor at $p$

(2) Suppose $T\left(e_{0}, \cdot\right)$ is dual to a future directed timelike vector $W$ at $p$. We have

$$
\inf _{\left(Y, T_{0}\right) \in \mathcal{P}} \lim _{r \rightarrow 0} r^{-3} E\left(\Sigma_{r}, Y_{r}, T_{0}\right)=\frac{4 \pi}{3} \sqrt{-\langle W, W\rangle} .
$$

The infimum is achieved by a unique $\left(A, C_{i}\right)$.

Proof. The quasi-local energy is

$\frac{1}{8 \pi} \int_{\Sigma}\left[f\left(V^{2}+V^{4}|\nabla \tau|^{2}\right)+\operatorname{div}\left(V^{2} \nabla \tau\right) \sinh ^{-1}\left(\frac{f \operatorname{div}\left(V^{2} \nabla \tau\right)}{\left|H_{0}\right||H|}\right)-\alpha_{H_{0}}\left(V^{2} \nabla \tau\right)+\alpha_{H}\left(V^{2} \nabla \tau\right)\right] d \Sigma$

where

$$
\begin{aligned}
f & =\frac{h_{0}^{(1)}-h^{(1)}}{A} r+O\left(r^{2}\right) \\
V^{2} & =A^{2}-\sum_{i} C_{i}^{2}+O(r) \\
V^{2} \nabla_{a} \tau & =r C_{i} \tilde{\nabla}_{a} \tilde{X}^{i}+O\left(r^{2}\right) .
\end{aligned}
$$

It is easy to see that

$$
E\left(\Sigma, Y, T_{0}\right)=\left(A e+C_{i} p^{i}\right) r^{3}+O\left(r^{4}\right)
$$

where

$$
\begin{gathered}
e=\frac{1}{8 \pi} \int_{\Sigma_{r}}\left(h_{0}^{(1)}-h^{(1)}\right) d S^{2} \\
p^{i}=\frac{1}{8 \pi} \int_{\Sigma_{r}} \tilde{X}^{i} \tilde{\nabla}^{a}\left(\alpha_{H}^{(2)}-\alpha_{H_{0}}^{(2)}\right)_{a} d S^{2} .
\end{gathered}
$$

It suffices to show that

$$
\begin{aligned}
\frac{1}{8 \pi} \int_{\Sigma_{r}}\left(h_{0}^{(1)}-h^{(1)}\right) d S^{2} & =\frac{4 \pi}{3} T\left(e_{0}, e_{0}\right) \\
\frac{1}{8 \pi} \int_{\Sigma_{r}} \tilde{X}^{i} \tilde{\nabla}^{a}\left(\alpha_{H}^{(2)}-\alpha_{H_{0}}^{(2)}\right)_{a} d S^{2} & =\frac{4 \pi}{3} T\left(e_{0}, e_{i}\right)
\end{aligned}
$$

For (7.1), we consider the Gauss curvature $K$ of $\Sigma_{r}$. It admits the following expansion

$$
2 \sqrt{K}=\frac{2}{r}+k^{(1)} r+O\left(r^{2}\right)
$$

From Lemma 4.1 of [13], we have

$$
\frac{1}{8 \pi} \int_{\Sigma_{r}}\left(k^{(1)}-h^{(1)}\right) d S^{2}=\frac{4 \pi}{3}\left(\bar{R} i c\left(e_{0}, e_{0}\right)+\frac{1}{2} \bar{R}\right)
$$


On the other hand, from the Gauss equation of image of the isometric embedding of $\Sigma_{r}$ in the hyperbolic space, we have

$$
K=-1+\frac{1}{4}\left|H_{0}\right|^{2}+O\left(r^{2}\right)
$$

and thus

$$
k^{(1)}=h_{0}^{(1)}-1
$$

As a result,

$$
\int_{\Sigma_{r}}\left(h_{0}^{(1)}-h^{(1)}\right) d S^{2}=\frac{4 \pi}{3}\left(\bar{R} i c\left(e_{0}, e_{0}\right)+\frac{1}{2} \bar{R}+3\right)=8 \pi T\left(e_{0}, e_{0}\right) .
$$

Next we compute $p^{i}$. From (6.4), we conclude that

$$
\int_{S^{2}} \tilde{X}^{i} \tilde{\nabla}^{a}\left(\alpha_{H_{0}}^{(2)}\right)_{a} d S^{2}=0 .
$$

Using the expansion for $d i v_{\sigma} \alpha_{H}$ from Lemma 5.3, we have

$$
\begin{aligned}
& p^{i}=r^{3} \int_{S^{2}}\left[\tilde{\Delta}\left[\frac{1}{2} \bar{R}_{L \underline{L} L \underline{L}}+\frac{1}{6} \bar{R} i c(L, L)+\frac{1}{3} \bar{R} i c(L, \underline{L})\right]\right. \\
& \left.-\bar{R}_{L \underline{L L} \underline{L}}-\frac{1}{3} \bar{R} i c(L, \underline{L})-\frac{1}{6} \bar{R} i c(L, L)\right] \tilde{X}^{i} d S^{2}+O\left(r^{4}\right) \\
& =r^{3} \int_{S^{2}}\left[-2 \bar{R}_{L \underline{L} L \underline{L}}-\bar{R} i c(L, \underline{L})-\frac{1}{2} \bar{R} i c(L, L)\right] \tilde{X}^{i} d S^{2}+O\left(r^{4}\right)
\end{aligned}
$$

where we apply integration by parts for the last equality.

To evaluate the above integral, we switch to the orthogonal frame $\left\{e_{0}, e_{i}\right\}$. We have

$$
\begin{aligned}
\int_{S^{2}}\left[-2 \bar{R}_{L \underline{L} L \underline{L}}-\bar{R} i c(L, \underline{L})-\frac{1}{2} \bar{R} i c(L, L)\right] \tilde{X}^{i} d S^{2} & =-\int_{S^{2}} \bar{R} i c\left(e_{0}, e_{j}\right) \tilde{X}^{j} \tilde{X}^{i} d S^{2} \\
& =-\frac{4 \pi}{3} \bar{R} i c\left(e_{0}, e_{i}\right) .
\end{aligned}
$$

This finishes the proof of part (1) since $\bar{R} i c\left(e_{0}, e_{i}\right)=8 \pi T_{0 i}$.

For part (2), we recall that from (2.2), we have

$$
A \geq \sqrt{1+|\vec{C}|^{2}} \geq 1
$$

Suppose $T\left(e_{0}, \cdot\right)$ is dual to a future directed timelike vector $V$. Fixing $\vec{C}, T\left(e_{0}, A e_{0}+C_{i} e_{i}\right)$ is strictly increasing in $A$ and the minimum of the quasi-local energy can only occur on the set of observers, $O$, such that

$$
A=\sqrt{1+|\vec{C}|^{2}}
$$

From here the Theorem easily follows. 
Remark 5. While the proof is similar to [12, Theorem 4.1] of the small sphere limit of the Wang-Yau quasi-local energy, The proof there used [31, Theorem 2.1] which evaluates the limit of the quasi-local energy under the compatibility condition of mean curvature

$$
\lim _{r \rightarrow 0} \frac{|H|}{\left|H_{0}\right|}=1 \text {. }
$$

However, we do not have the corresponding result when the AdS space is used as the reference. It will be interesting to establish the analog of [31, Theorem 2.1] for the newly defined quasi-local energy.

\section{SMALl SPhere Limit of THE QUASI-LOCAL ENERGY IN VACUUM SPACETIMES}

In this section, we begin the computation of the small sphere limit of the quasi-local energy in vacuum spacetimes. First, we derive the following lemma about the Gauss curvature of $\Sigma_{r}$.

Lemma 8.1. For vacuum spacetimes, the Gauss curvature $K$ of $\Sigma_{r}$ admits the following expansion

$$
K=\frac{1}{r^{2}}+2 \rho+O(r)
$$

Proof. Recall that the induced metric $\sigma$ is

$$
\sigma_{a b}=r^{2} \tilde{\sigma}_{a b}+\frac{r^{4}}{3} \alpha_{a b}+O\left(r^{5}\right)
$$

and $\alpha_{a b}$ is traceless. As a result, we have

$$
K=\frac{1}{r^{2}}+\frac{1}{6} \tilde{\nabla}^{a} \tilde{\nabla}^{b} \alpha_{a b}+O(r)
$$

The lemma now follows from Lemma 5.6.

Next, we solve the optimal embedding equation derived in Lemma 6.2.

Lemma 8.2. For the observer $T_{0}=(A, \vec{B}, \vec{C}, \vec{D})$, the solution of the optimal embedding equation gives

$$
Y_{0}^{(3)}=-\frac{1}{3} W_{0}+\frac{\sum_{i} C_{i} P_{i}}{A} .
$$

Proof. Recall that for the vacuum spacetime, we have

$$
|H|=\frac{2}{r}+\left(W_{0}+1\right) r+O\left(r^{2}\right) .
$$

On the other hand, for $\left|H_{0}\right|$, it suffices to compute the mean curvature of the isometric embedding into the hyperbolic space. From the Gauss equation of the surface into the hyperbolic space, we conclude that

$$
K=-1+\frac{1}{4}\left|H_{0}\right|^{2}+O\left(r^{2}\right)
$$


As a result, from the above lemma, we have

$$
\left|H_{0}\right|=\frac{2}{r}+\left(2 W_{0}+1\right) r+O\left(r^{2}\right)
$$

Hence,

$$
f^{(1)}=\frac{W_{0}}{A} .
$$

The rest of the proof is the same as Lemma 6.3 of [13].

For each $T_{0}$, we shall compute $8 \pi E\left(\Sigma_{r}, Y_{r}\left(T_{0}\right), T_{0}\right)$ which is given by

$$
\begin{aligned}
& \int_{\Sigma_{r}} f\left(V^{2}+V^{4}|\nabla \tau|^{2}\right)+\left(\operatorname{div} V^{2} \nabla \tau\right) \sinh ^{-1}\left(\frac{f d i v V^{2} \nabla \tau}{|H|\left|H_{0}\right|}\right) d \Sigma_{r} \\
& -\int_{\Sigma_{r}} \alpha_{H_{0}}\left(V^{2} \nabla \tau\right) d \Sigma_{r}+\int_{\Sigma_{r}} \alpha_{H}\left(V^{2} \nabla \tau\right) d \Sigma_{r} .
\end{aligned}
$$

We evaluate the three integrals in the next three sections, respectively and put the results together in Section 12 .

\section{THE ENERGY COMPONENT}

In this section, we evaluate the first integral in (8.1):

$$
\int_{\Sigma_{r}} f\left(V^{2}+V^{4}|\nabla \tau|^{2}\right)+\operatorname{div}\left(V^{2} \nabla \tau\right) \sinh ^{-1}\left(\frac{f \operatorname{div}\left(V^{2} \nabla \tau\right)}{|H|\left|H_{0}\right|}\right) d \Sigma_{r}
$$

It suffices to evaluate $\int_{\Sigma_{r}} f\left[V^{2}+V^{4}|\nabla \tau|^{2}+\frac{\left(\operatorname{div}\left(V^{2} \nabla \tau\right)\right)^{2}}{|H|\left|H_{0}\right|}\right] d \Sigma_{r}$ since for $x$ small,

$$
\sinh ^{-1}(x)=x+O\left(x^{3}\right) \text {. }
$$

Denote the expansion of the physical data by

$$
\begin{aligned}
& \sigma_{a b}=r^{2} \tilde{\sigma}_{a b}+r^{4} \sigma_{a b}^{(4)}+r^{5} \sigma_{a b}^{(5)}+O\left(r^{6}\right) \\
& |H|=\frac{2}{r}+r h^{(1)}+r^{2} h^{(2)}+r^{3} h^{(3)}+O\left(r^{4}\right) \\
& \alpha_{H}=r^{2} \alpha_{H}^{(2)}+r^{3} \alpha_{H}^{(3)}+r^{4} \alpha_{H}^{(4)}+O\left(r^{5}\right) .
\end{aligned}
$$

Similarly, we have

$$
\begin{aligned}
& \left|H_{0}\right|=\frac{2}{r}+r h_{0}^{(1)}+r^{2} h_{0}^{(2)}+r^{3} h_{0}^{(3)}+O\left(r^{4}\right) \\
& \alpha_{H_{0}}=r^{2} \alpha_{H_{0}}^{(2)}+r^{3} \alpha_{H_{0}}^{(3)}+r^{4} \alpha_{H_{0}}^{(4)}+O\left(r^{5}\right) .
\end{aligned}
$$

First we derive the following lemma. 


\section{Lemma 9.1.}

$$
\begin{aligned}
V^{2}= & \left(A^{2}-\sum_{i} C_{i}^{2}\right)+2\left(A B_{i} \tilde{X}^{i}-\sum_{i} C_{i} D_{p} \epsilon_{p q i} \tilde{X}^{q}\right) r \\
& +r^{2}\left[\left(B_{i} \tilde{X}^{i}\right)^{2}+\left(C_{i} \tilde{X}^{i}\right)^{2}-\sum_{i}\left(D_{p} \epsilon_{p q i} \tilde{X}^{q}\right)^{2}+\left(A^{2}-\sum_{i} C_{i}^{2}\right)\right]+O\left(r^{3}\right) \\
V^{4}|\nabla \tau|^{2}= & C_{i} C_{j}\left(\delta^{i j}-\tilde{X}^{i} \tilde{X}^{j}\right)+2 r \sum_{i} C_{i} D_{p} \epsilon_{p q i} \tilde{X}^{q} \\
& +\left(\left(\delta^{i j}-\tilde{X}^{i} \tilde{X}^{j}\right) C_{i} C_{j}+\sum_{i}\left(D_{p} \epsilon_{p q i} \tilde{X}^{q}\right)^{2}+g_{1}\right) r^{2}+O\left(r^{3}\right) \\
\left(\operatorname{div}\left(V^{2} \nabla \tau\right)\right)^{2}= & 4 C_{i} C_{j}\left(\tilde{X}^{i} \tilde{X}^{j}\right) r^{-2}+\left(4 C_{i} C_{j} \tilde{X}^{i} \tilde{X}^{j}+g_{2}\right)+O(r),
\end{aligned}
$$

where

$$
\begin{aligned}
& g_{1}=\sum_{i, j} C_{i} C_{j}\left(R_{i j}+2 \tilde{\nabla} \tilde{X}^{i} \tilde{\nabla} Y_{j}^{(3)}\right)+2 A C_{i} \tilde{\nabla} \tilde{X}^{i} \tilde{\nabla} Y_{0}^{(3)} \\
& g_{2}=\sum_{i, j} 4 C_{i} C_{j} \tilde{X}^{i}\left(S_{j}-\tilde{\Delta} X_{j}^{(3)}\right)-4 A C_{i} \tilde{X}^{i} \tilde{\Delta} Y_{0}^{(3)},
\end{aligned}
$$

and $R_{i j}$ and $S_{j}$ are defined in (5.31).

Proof. We have

$$
V^{2}=-\left\langle T_{0}, T_{0}\right\rangle=\left(A Y^{0}+C_{i} Y^{i}\right)^{2}+\left(A Y^{4}+B_{i} Y^{i}\right)^{2}-\sum_{i}\left(C_{i} Y^{4}+B_{i} Y^{0}+D_{p} \epsilon_{p q i} Y^{q}\right)^{2}
$$

where $Y^{0}=O\left(r^{3}\right), Y^{i}=r \tilde{X}^{i}+O\left(r^{3}\right)$ and $Y^{4}=\sqrt{1+r^{2}}+O\left(r^{3}\right)$. This gives the expansion for $V^{2}$.

For the other two terms, we have

$$
V^{2} \nabla \tau=-T_{0}^{\perp}=\left(A Y^{0}+C_{i} Y^{i}\right) \nabla Y^{4}+\left(A Y^{4}+B_{i} Y^{i}\right) \nabla Y^{0}+\left(C_{i} Y^{4}+B_{i} Y^{0}+D_{p} \epsilon_{p q i} Y^{q}\right) \nabla Y^{i} .
$$

As a result,

$$
\begin{aligned}
V^{4}|\nabla \tau|^{2}= & \left(C_{i} Y^{4}+B_{i} Y^{0}+D_{p} \epsilon_{p q i} Y^{q}\right)\left(C_{j} Y^{4}+B_{j} Y^{0}+D_{m} \epsilon_{m n j} Y^{n}\right)\left(\delta^{i j}-\tilde{X}^{i} \tilde{X}^{j}\right) \\
& +r^{2}\left[\sigma^{(0) a b}\left(C_{i} \tilde{X}_{a}^{i}\right)\left(C_{j} \tilde{X}_{b}^{j}\right)+2\left(C_{i} \tilde{\nabla} \tilde{Y}^{i}\right)\left(C_{j} \tilde{\nabla} Y_{j}^{(3)}+A \tilde{\nabla} Y_{0}^{(3)}\right)\right]+O\left(r^{3}\right),
\end{aligned}
$$

and the formula follows from (5.31) and (6.1). Similarly,

$$
\begin{aligned}
\left(\operatorname{div} V^{2} \nabla \tau\right)^{2}= & 4 \sum_{i j}\left(C_{i} Y^{4}+B_{i} Y^{0}+D_{p} \epsilon_{p q i} Y^{q}\right)\left(C_{j} Y^{4}+B_{j} Y^{0}+D_{m} \epsilon_{m n j} Y^{n}\right)\left(\tilde{X}^{i} \tilde{X}^{j}\right) r^{-2} \\
& +4\left(C_{i} \tilde{X}^{i}\right)\left(C_{j} \tilde{\sigma}^{a b} \gamma_{a b}^{(2) c} \tilde{X}_{c}^{j}\right)-4\left(C_{i} \tilde{X}^{i}\right)\left(C_{j} \tilde{\Delta} Y_{j}^{(3)}+A \tilde{\Delta} Y_{0}^{(3)}\right)+O(r),
\end{aligned}
$$


where

$$
\begin{aligned}
& 4\left(C_{i} \tilde{X}^{i}\right)\left(C_{j} \tilde{\sigma}^{a b} \gamma_{a b}^{(2) c} \tilde{X}_{c}^{j}\right)-4\left(C_{i} \tilde{X}^{i}\right)\left(C_{j} \tilde{\Delta} Y_{j}^{(3)}+A \tilde{\Delta} Y_{0}^{(3)}\right) \\
= & 4 C_{i} C_{j} \tilde{X}^{i} S_{j}-4\left(C_{i} \tilde{X}^{i}\right)\left(C_{j} \tilde{\Delta} Y_{j}^{(3)}+A \tilde{\Delta} Y_{0}^{(3)}\right) \\
= & 4 C_{i} C_{j} \tilde{X}^{i}\left(S_{j}-\tilde{\Delta} Y_{j}^{(3)}\right)-4 A C_{i} \tilde{X}^{i} \tilde{\Delta} Y_{0}^{(3)}
\end{aligned}
$$

With the above lemma, we compute $f\left[V^{2}+V^{4}|\nabla \tau|^{2}+\frac{\left(\operatorname{div}\left(V^{2} \nabla \tau\right)\right)^{2}}{|H|\left|H_{0}\right|}\right]$.

\section{Lemma 9.2.}

$$
\begin{aligned}
& f\left[V^{2}+V^{4}|\nabla \tau|^{2}+\frac{\left(\operatorname{div}\left(V^{2} \nabla \tau\right)\right)^{2}}{|H|\left|H_{0}\right|}\right] \\
= & A r\left(h_{0}^{(1)}-h^{(1)}\right)+r^{2}\left[A\left(h_{0}^{(2)}-h^{(2)}\right)+\frac{W_{0} B_{i} \tilde{X}^{i}}{A}\right]+ \\
& A r^{3}\left[\left(h_{0}^{(3)}-h^{(3)}\right)+\frac{\left(h_{0}^{(2)}-h^{(2)}\right) B_{i} \tilde{X}^{i}}{A^{2}}+\frac{\left(W_{0}\right)\left[g_{1}+\frac{g_{2}}{4}-\frac{3 W_{0}}{2} C_{i} C_{j} \tilde{X}^{i} \tilde{X}^{j}\right]}{2 A^{2}}\right]+O\left(r^{4}\right) .
\end{aligned}
$$

Proof. From Lemma 9.1, we have

$$
\begin{aligned}
& V^{2}+V^{4}|\nabla \tau|^{2}+\frac{\left(d i v V^{2} \nabla \tau\right)^{2}}{\left|H_{0}\right|^{2}} \\
= & A^{2}+2 A B_{i} \tilde{X}^{i} r \\
& +r^{2}\left[\left(B_{i} \tilde{X}^{i}\right)^{2}+A^{2}+\left(C_{i} \tilde{X}^{i}\right)^{2}+g_{1}+\frac{g_{2}}{4}-h_{0}^{(1)} \sum_{i j} C_{i} C_{j} \tilde{X}^{i} \tilde{X}^{j}\right],
\end{aligned}
$$

and thus

$$
\begin{aligned}
& \left|H_{0}\right| \sqrt{V^{2}+V^{4}|\nabla \tau|^{2}+\frac{\left(d i v V^{2} \nabla \tau\right)^{2}}{\left|H_{0}\right|^{2}}} \\
= & A\left[\frac{2}{r}+\frac{2 A B_{i} \tilde{X}^{i}}{A^{2}}+r\left(h_{0}^{(1)}+\frac{A^{2}+\left(C_{i} \tilde{X}^{i}\right)^{2}+g_{1}+\frac{g_{2}}{4}-h_{0}^{(1)} \sum_{i j} C_{i} C_{j} \tilde{X}^{i} \tilde{X}^{j}}{A^{2}}\right)\right]+O\left(r^{2}\right) .
\end{aligned}
$$

We can compute $|H| \sqrt{1+|\nabla \tau|^{2}+\frac{(\Delta \tau)^{2}}{|H|^{2}}}$ and $1+|\nabla \tau|^{2}+\frac{(\Delta \tau)^{2}}{\left|H_{0}\right||H|}$ similarly. As a result, $f\left(1+|\nabla \tau|^{2}+\frac{(\Delta \tau)^{2}}{|H|\left|H_{0}\right|}\right)$ is equal to

$$
\begin{aligned}
& \left(\frac{4}{r}+\left(h_{0}^{(1)}+h^{(1)}\right) r\right)\left[\left(h_{0}^{(1)}-h^{(1)}\right) r+\left(h_{0}^{(2)}-h^{(2)}\right) r^{2}+\left(h_{0}^{(3)}-h^{(3)}\right) r^{3}\right] \times \\
& \frac{A^{2}+B_{i} \tilde{X}^{i} r+r^{2}\left[\left(C_{i} \tilde{X}^{i}\right)^{2}+g_{1}+\frac{g_{2}}{4}-\frac{\left(h_{0}^{(1)}+h^{(1)}\right)}{2} C_{i} C_{j} \tilde{X}^{i} \tilde{X}^{j}\right]}{A\left\{\frac{4}{r}+\frac{4 B_{i} \tilde{X}^{i}}{A^{2}}+r\left[2\left(C_{i} \tilde{X}^{i}\right)^{2}+h_{0}^{(1)}+h^{(1)}+\frac{2 g_{1}+\frac{g_{2}}{2}-2\left(h_{0}^{(1)}+h^{(1)}\right) C_{i} C_{j} \tilde{X}^{i} \tilde{X}^{j}}{A^{2}}\right]\right\}} .
\end{aligned}
$$


Finally we plug in $h_{0}^{(1)}=2 W_{0}+1$ and $h^{(1)}=W_{0}+1$.

\section{Lemma 9.3.}

$$
\begin{aligned}
& \lim _{r \rightarrow 0} r^{-5} \int_{\Sigma_{r}} f\left[V^{2}+V^{4}|\nabla \tau|^{2}+\frac{\left(\operatorname{div}\left(V^{2} \nabla \tau\right)\right)^{2}}{|H|\left|H_{0}\right|}\right] d \Sigma_{r} \\
= & A \int_{S^{2}}\left(h_{0}^{(3)}-h^{(3)}\right) d S^{2}-\frac{3 C_{i} C_{j}}{4 A} \int_{S^{2}} W_{0}^{2} \tilde{X}^{i} \tilde{X}^{j} d S^{2} \\
& +\frac{C_{i} C_{j}}{2 A} \int_{S^{2}}\left(W_{0}\right)\left[R_{i j}+2 \tilde{\nabla} \tilde{X}^{i} \cdot \tilde{\nabla}\left(Y_{j}^{(3)}+P_{j}\right)+\tilde{X}^{i}\left(S_{j}-\tilde{\Delta} Y_{j}^{(3)}+12 P_{j}\right)\right] d S^{2}
\end{aligned}
$$

Proof. For the volume form, we have $d \Sigma_{r}=r^{2} d S^{2}+O\left(r^{5}\right)$ from the expansion of metric in Lemma 5.3. As a result, it suffices to use $r^{2} d S^{2}$ for the volume form.

For the mean curvature in AdS space,

$$
\left|H_{0}\right|=2 \sqrt{K+1}+O\left(r^{3}\right)
$$

since $Y_{0}=O\left(r^{3}\right)$. Using the Gauss equation of the surface in $N$, we conclude that

$$
\left|H_{0}\right|-|H|=\rho r+D \rho r^{2}+O\left(r^{3}\right)
$$

That is

$$
\begin{aligned}
& \left(h_{0}^{(1)}-h^{(1)}\right)=\rho \\
& \left(h_{0}^{(2)}-h^{(2)}\right)=D \rho
\end{aligned}
$$

It follows that for $i=1,2$

$$
\int_{S^{2}}\left(h_{0}^{(i)}-h^{(i)}\right) d S^{2}=0 .
$$

Moreover, for parity reason, it is easy to see that

$$
\int_{S^{2}} W_{0} \tilde{X}^{i} d S^{2}=0
$$

Hence, we are left with only the $O\left(r^{5}\right)$ terms. We compute

$$
\begin{aligned}
\int_{S^{2}} W_{0}\left(g_{1}+\frac{g_{2}}{4}\right) d S^{2}= & C_{i} C_{j} \int_{S^{2}} W_{0}\left[R_{i j}+2 \tilde{\nabla} \tilde{X}^{i} \tilde{\nabla}\left(Y_{j}^{(3)}+P_{j}\right)+\tilde{X}^{i}\left(S_{j}-\tilde{\Delta} Y_{j}^{(3)}+12 P_{j}\right)\right] d S^{2} \\
& -A C_{j} \int_{S^{2}} W_{0}\left(\frac{2}{3} \tilde{\nabla} \tilde{X}^{i} \tilde{\nabla} W_{0}+2 \tilde{X}^{i} W_{0}\right) d S^{2} .
\end{aligned}
$$

Due to parity, we have

$$
\int_{S^{2}} W_{0}\left(\frac{2}{3} \tilde{\nabla} \tilde{X}^{i} \tilde{\nabla} W_{0}+2 \tilde{X}^{i} W_{0}\right) d S^{2}=0 .
$$

Finally, from Lemma 5.6, we have

$$
\int_{S^{2}} D \rho \tilde{X}^{i} d S^{2}=\frac{1}{20} \int_{S^{2}} \tilde{\nabla}^{a} \tilde{\nabla}^{b} D \alpha_{a b} \tilde{X}^{i} d S^{2}=-\frac{1}{20} \int_{S^{2}} D \alpha_{a b} \tilde{\sigma}^{a b} \tilde{X}^{i} d S^{2}=0 .
$$


Namely,

$$
\int_{S^{2}}\left(h_{0}^{(2)}-h^{(2)}\right) \tilde{X}^{i} d S^{2}=0 .
$$

The lemma follows from Lemma 9.2.

9.1. Computation of $\int\left(h_{0}^{(3)}-h^{(3)}\right)$. Suppose $Y$ is the isometric embedding of $\sigma$ into the AdS space such that

$$
\begin{aligned}
& Y_{0}=\sum_{i=3}^{\infty} Y_{0}^{(i)} r^{i} \\
& Y_{k}=r \tilde{X}^{k}+\sum_{i=3}^{\infty} Y_{k}^{(i)} r^{i} \\
& Y_{4}=\sqrt{1+\sum_{i} Y_{i}^{2}-Y_{0}^{2}}
\end{aligned}
$$

where $Y_{0}^{(3)}$ and $Y_{i}^{(3)}$ are given by Lemma 6.1 and Lemma 8.2, respectively.

Let $Y^{\prime}$ be the isometric embedding of $\sigma$ into the hyperbolic space in the AdS space where

$$
\begin{gathered}
\left(Y_{0}\right)^{\prime}=0 \\
\left(Y_{i}\right)^{\prime}=r \tilde{X}^{i}+r^{3} Y_{i}^{\prime(3)}+r^{4} Y_{i}^{\prime(4)}+r^{5} Y_{i}^{\prime(5)}+O\left(r^{6}\right) .
\end{gathered}
$$

Let $A^{\prime}$ be the second fundamental form the embedding $Y^{\prime}$ in the hyperbolic space and $\AA^{\prime}$ be its traceless part.

$$
\stackrel{\circ}{A}_{a b}^{\prime}=r^{3} \stackrel{\circ}{A}_{a b}^{\prime(3)}+O\left(r^{4}\right)
$$

Suppose the Gauss curvature $K$ of $\sigma$ has the following expansion:

$$
2 \sqrt{K}=\frac{2}{r}+k^{(1)} r+k^{(2)} r^{2}+k^{(3)} r^{3}+O\left(r^{4}\right) .
$$

We have

Proposition 9.1. The integral $\int_{S^{2}}\left(h_{0}^{(3)}-h^{(3)}\right) d S^{2}$ can be written as follows:

$$
\begin{aligned}
\int_{S^{2}}\left(h_{0}^{(3)}-h^{(3)}\right) d S^{2}= & \frac{1}{2} \int_{S^{2}}\left|A^{\prime}(3)\right|_{\tilde{\sigma}}^{2} d S^{2}+\int\left(k^{(3)}-h^{(3)}-\frac{1}{4}\right) d S^{2}-\frac{2}{3} \int_{S^{2}} W_{0}^{2} d S^{2} \\
& -30 \frac{C_{i} C_{j}}{A^{2}} \int_{S^{2}} P_{i} P_{j} d S^{2} .
\end{aligned}
$$

Proof. We first rewrite

$$
\int_{\Sigma_{r}}\left(\left|H_{0}\right|-|H|\right) d \Sigma_{r}=\int_{\Sigma_{r}}\left(\left|H_{0}\right|-2 \sqrt{K+1}\right) d \Sigma_{r}+\int_{\Sigma_{r}}(2 \sqrt{K+1}-|H|) d \Sigma_{r} .
$$

We have

$$
\int_{\Sigma_{r}}(2 \sqrt{K+1}-|H|) d \Sigma_{r}=r^{5} \int_{S^{2}}\left(k^{(3)}-h^{(3)}-\frac{1}{4}\right) d S^{2}+O\left(r^{6}\right) .
$$


To evaluate $\int_{\Sigma_{r}}\left(\left|H_{0}\right|-2 \sqrt{K+1}\right) d \Sigma_{r}$, recall that $\left|H_{0}\right|^{2}$ is given by

$$
\left|H_{0}\right|^{2}=-\left(\Delta Y_{0}\right)^{2}-\left(\Delta Y_{4}\right)^{2}+\sum_{i=1}^{3}\left(\Delta Y_{i}\right)^{2}+4
$$

since the AdS space is an umbilical hypersurface in $\mathbb{R}^{3,2}$.

Let $H_{0}^{\prime}$ be the mean curvature of $Y^{\prime}$. Similarly, $\left|H_{0}^{\prime}\right|$ is given by

$$
\left|H_{0}^{\prime}\right|^{2}=-\left(\Delta Y_{4}^{\prime}\right)^{2}+\sum_{i=1}^{3}\left(\Delta\left(Y_{i}\right)^{\prime}\right)^{2}+4
$$

The Gauss equation of $Y^{\prime}(\Sigma)$ in the hyperbolic space reads

$$
4 K=\left(H_{0}^{\prime}\right)^{2}-4-2\left|\AA^{\prime}\right|^{2} .
$$

We compute from (9.2), (9.4), and (9.3) that

$$
\left|H_{0}\right|^{2}-4 K-4=2\left|\AA^{\prime}\right|^{2}-\left(\Delta Y_{0}\right)^{2}-\left(\Delta Y_{4}\right)^{2}+\left(\Delta Y_{4}^{\prime}\right)^{2}+\sum_{i=1}^{3}\left(\Delta Y_{i}\right)^{2}-\sum_{i=1}^{3}\left(\Delta Y_{i}^{\prime}\right)^{2},
$$

where

$$
\Delta Y_{0}=\Delta\left(r^{3} Y_{0}^{(3)}+O\left(r^{4}\right)\right)=r \tilde{\Delta} Y_{0}^{(3)}+O\left(r^{2}\right)
$$

$\Delta Y_{4}=O\left(r^{2}\right), \Delta Y_{4}^{\prime}=O\left(r^{2}\right)$ and

$$
\begin{aligned}
\sum_{i=1}^{3}\left(\Delta Y_{i}\right)^{2}-\sum_{i=1}^{3}\left(\Delta\left(Y_{i}\right)^{\prime}\right)^{2} & =\sum_{i=1}^{3} \Delta\left(Y_{i}-Y_{i}^{\prime}\right) \Delta\left(Y_{i}+Y_{i}^{\prime}\right) \\
& =-4 r^{2} \tilde{X}^{i} \tilde{\Delta}\left(Y_{i}^{(5)}-Y_{i}^{\prime(5)}\right)+O\left(r^{3}\right) .
\end{aligned}
$$

As a result, we have

$$
\begin{aligned}
& \int_{S^{2}}\left(h_{0}^{(3)}-h^{(3)}\right) d S^{2} \\
= & \frac{1}{2} \int_{S^{2}}\left|\stackrel{A}{ }^{\prime}(3)\right|_{\tilde{\sigma}}^{2} d S^{2}-\frac{1}{4} \int_{S^{2}}\left(\tilde{\Delta} Y_{0}^{(3)}\right)^{2} d S^{2}-\int_{S^{2}} \tilde{X}^{i} \tilde{\Delta}\left(Y_{i}^{(5)}-Y_{i}^{\prime(5)}\right) d S^{2}+\int_{S^{2}}\left(k^{(3)}-\frac{1}{4}-h^{(3)}\right) d S^{2} .
\end{aligned}
$$

To evaluate the second last terms, we need

Lemma 9.4. If we choose $Y_{i}^{(3)}=Y_{i}^{\prime(3)}$ and $Y_{i}^{(4)}=Y_{i}^{\prime(4)}$, then $Y_{i}^{(5)}$ and $Y_{i}^{\prime(5)}$ are related by

$$
2 \tilde{\nabla} \tilde{X}^{i} \cdot \tilde{\nabla}\left(Y_{i}^{(5)}-Y_{i}^{\prime(5)}\right)=\left|\tilde{\nabla} Y_{0}^{(3)}\right|^{2} .
$$

Proof. This follows directly from the expansion of the metric and the isometric embedding equation.

The proposition now follows from the expression of $Y_{0}^{(3)}$ in Lemma 8.2 .

The traceless part $\stackrel{\circ}{A b}_{a b}$ of $h_{a b}$ has the following expansion

$$
\stackrel{\circ}{A}_{a b}=r^{3} \stackrel{\circ}{A}_{a b}^{(3)}+O\left(r^{4}\right) .
$$


Lemma 9.5.

$$
\AA_{a b}^{(3)}=\left(\tilde{X}_{a}^{i} \tilde{X}_{b}^{j}+\tilde{X}_{b}^{i} \tilde{X}_{a}^{j}\right)\left(-\frac{1}{4} W_{0} \delta_{i j}-\frac{1}{2} W_{0 i 0 j}\right) .
$$

Remark 6. The proof is the same as Lemma 7.5 of [12] by embedding everything in $\mathbb{R}^{3,2}$ as above.

As result, from Lemma 7.6 of [12], we conclude again that

\section{Lemma 9.6.}

$$
\int_{S^{2}}\left|\AA^{(3)}\right|_{\tilde{\sigma}}^{2} d S^{2}=3 \int_{S^{2}} W_{0}^{2} d S^{2}
$$

9.1.1. Computing $\int\left(k^{(3)}-\frac{1}{4}-h^{(3)}\right) d S^{2}$.

\section{Lemma 9.7.}

$$
\int_{S^{2}}\left(k^{(3)}-\frac{1}{4}-h^{(3)}\right) d S^{2}=-\frac{3}{4} \int_{S^{2}} W_{0}^{2} d S^{2}-\frac{1}{60} \int_{S^{2}}|\alpha|^{2} d S^{2}+\frac{11}{45} \int_{S^{2}}|\beta|^{2} d S^{2} .
$$

Proof. First, we compute $\int k^{(3)} d S^{2}$. From (9.1), we have

$$
K=\frac{1}{r^{2}}+k^{(1)}+k^{(2)} r+\left[k^{(3)}+\frac{\left(k^{(1)}\right)^{2}}{4}\right] r^{2}+O\left(r^{3}\right) .
$$

We also have

$$
d \Sigma_{r}=\left(r^{2}-\frac{1}{180} r^{6}|\alpha|^{2}\right) d S^{2}+O\left(r^{7}\right)
$$

from the expansion of $\sigma^{a b} l_{a b}$ in Lemma[5.8. By the Gauss-Bonnet theorem $\int_{\Sigma_{r}} K d \Sigma_{r}=4 \pi$. Collecting the $O\left(r^{4}\right)$ terms from the left hand side, we have

$$
\int_{S^{2}} k^{(3)}+\frac{\left(k^{(1)}\right)^{2}}{4} d S^{2}=\frac{1}{180} \int_{S^{2}}|\alpha|^{2} d S^{2}
$$

Furthermore, $k^{(1)}=2 W_{0}$. Hence

$$
\int_{S^{2}} k^{(3)} d S^{2}=-\int_{S^{2}} W_{0}^{2} d S^{2}+\frac{1}{180} \int_{S^{2}}|\alpha|^{2} d S^{2}
$$

For $h^{(3)}$, we have

$$
h^{(3)}=\left(\sigma^{a b} n_{a b}\right)^{(3)}-\frac{1}{90}|\alpha|^{2}-\frac{\left(\sigma^{a b} n_{a b}^{(1)}\right)^{2}}{4} .
$$

Using Lemma 5.8 and Lemma 5.6, we conclude

$$
\begin{aligned}
& \int_{S^{2}}\left(k^{(3)}-\frac{1}{4}-h^{(3)}\right) d S^{2} \\
= & -\frac{3}{4} \int_{S^{2}} W_{0}^{2} d S^{2}+\frac{1}{60} \int_{S^{2}}|\alpha|^{2} d S^{2}-\int_{S^{2}}\left(\sigma^{a b} n_{a b}\right)^{(3)} d S^{2}+\int_{S^{2}} \frac{W_{0}}{2} d S^{2} \\
= & -\frac{3}{4} \int_{S^{2}} W_{0}^{2} d S^{2}-\frac{1}{60} \int_{S^{2}}|\alpha|^{2} d S^{2}+\frac{11}{45} \int_{S^{2}}|\beta|^{2} d S^{2} .
\end{aligned}
$$




\section{Computing the Reference Hamiltonian}

In this section, we compute the limit of the second integral in equation (8.1):

$$
\int_{\Sigma_{r}}-\alpha_{H_{0}}\left(V^{2} \nabla \tau\right) d \Sigma_{r}
$$

First we prove the following lemma about $\alpha_{H_{0}}$.

Lemma 10.1. Let $Y$ be an isometric embedding of $\Sigma_{r}$ into the AdS sapce such that

$$
Y^{0}=r^{3} Y_{0}^{(3)}+r^{4} Y_{0}^{(4)}+O\left(r^{5}\right) .
$$

We have

$$
\left(\alpha_{H_{0}}\right)_{a}=\frac{r^{2}}{2} \tilde{\nabla}_{a}(\tilde{\Delta}+2) Y_{0}^{(3)}+\frac{r^{3}}{2} \tilde{\nabla}_{a}(\tilde{\Delta}+2) Y_{0}^{(4)}+O\left(r^{4}\right)
$$

and

$$
\begin{aligned}
d i v_{\sigma} \alpha_{H_{0}}= & \frac{1}{2}\left(r^{-2} \Delta\right)\left(r^{-2} \Delta+2\right)\left(Y_{0}^{(3)}+r Y_{0}^{(4)}+r^{2} Y_{0}^{(5)}\right)-\frac{r^{2}}{2}\left(\tilde{\Delta}(\tilde{\Delta}-1) Y_{0}^{(3)}\right)+ \\
& r^{2}\left[\tilde{\sigma}^{a c} \tilde{\sigma}^{b d} \AA_{c d}^{\prime(3)} \tilde{\nabla}_{b} \tilde{\nabla}_{a} Y_{0}^{(3)}+Y_{0} \tilde{\Delta} Y_{0}^{(3)}+2 \tilde{\nabla} W_{0} \tilde{\nabla} Y_{0}^{(3)}-\frac{1}{2} \tilde{\Delta}\left(W_{0} \tilde{\Delta} Y_{0}^{(3)}\right)\right]+O\left(r^{3}\right) .
\end{aligned}
$$

Proof. We may assume, for this lemma alone that $T_{0}=\frac{\partial}{\partial t}$ since the qualities depends on $Y$ but not on $T_{0}$. (10.1) follows from the formula in the proof of Theorem 6.2 of [13] by treating the image of the isometric embedding as a small perturbation of the isometric embedding into the hyperbolic space. For (10.2), we use Theorem 5.2 of [13] that a surface in the AdS space is always a critical point of the quasi-local energy with respect to other isometric embeddings into the AdS space. That is, we consider a family of isometric embedding $Y(s)$ such that $Y(0)=Y$, we have

$$
\left.\frac{d}{d s}\right|_{s=0} E\left(\Sigma, Y(s), \frac{\partial}{\partial t}\right)=0
$$

As a result, if we consider

$$
\mathfrak{H}_{1}=\int V \widehat{H} d \widehat{\Sigma}
$$

and

$$
\begin{aligned}
\mathfrak{H}_{2} & =\int\left[\sqrt{\left(1+V^{2}|\nabla \tau|^{2}\right)\left|H_{0}\right|^{2} V^{2}+\operatorname{div}\left(V^{2} \nabla \tau\right)^{2}}\right. \\
& \left.-\operatorname{div}\left(V^{2} \nabla \tau\right) \sinh ^{-1} \frac{\operatorname{div}\left(V^{2} \nabla \tau\right)}{V\left|H_{0}\right| \sqrt{1+V^{2}|\nabla \tau|^{2}}}-V^{2} \alpha_{H_{0}}(\nabla \tau)\right] d \Sigma
\end{aligned}
$$

Then

$$
\left.\frac{d}{d s}\right|_{s=0} \mathfrak{H}_{1}=\left.\frac{d}{d s}\right|_{s=0} \mathfrak{H}_{2},
$$


where for the variation of $\mathfrak{H}_{2}$, it is understood that $H_{0}$ and $\alpha_{H_{0}}$ are fixed at their values at the initial surface $Y(0)$. On $Y(0)$, we have

$$
V=\sqrt{1+r^{2}}+O\left(r^{4}\right)
$$

We consider a family of isometric embedding such that

and

$$
\left.\frac{d}{d s}\right|_{s=0} Y^{0}=O\left(r^{3}\right)
$$

We conclude that

$$
Z^{i}=\left.\frac{d}{d s}\right|_{s=0} Y^{i}=O\left(r^{5}\right)
$$

and

$$
\left.\frac{d}{d s}\right|_{s=0} Y^{4}=O\left(r^{6}\right)
$$

$$
\left.\frac{d}{d s}\right|_{s=0} V=O\left(r^{6}\right)
$$

In terms of the static coordinate, we have

$$
y^{0}=\sqrt{1+r^{2}} t+O\left(r^{6}\right)
$$

and

$$
Y^{0}=\sqrt{1+r^{2}} \tau+O\left(r^{6}\right)
$$

Let

$$
\begin{gathered}
\delta \tau=\left.\frac{d}{d s}\right|_{s=0} \tau \\
(\delta \hat{\sigma})_{a b}=\left(1+r^{2}\right)\left(\partial_{a} \tau \partial_{b} \delta \tau+\partial_{b} \tau \partial_{a} \delta \tau\right)+O\left(r^{10}\right)
\end{gathered}
$$

and $Z^{i}$ satisfies the linearized isometric embedding equation

$$
\sum_{i} \partial_{a} Y^{i} \partial_{b} Z^{i}+\partial_{b} Y^{i} \partial_{a} Z^{i}=\left(1+r^{2}\right)\left(\partial_{a} \tau \partial_{b} \delta \tau+\partial_{b} \tau \partial_{a} \delta \tau\right)+O\left(r^{10}\right)
$$

Decomposing $Z$ into the tangential part $P_{a}$ and normal part $f e_{3}$ to the surface $\widehat{\Sigma}$ From equation (5.6) of [13], we have

$$
\delta \widehat{H}+\frac{1}{2} \hat{h}^{a b}(\delta \hat{\sigma})_{a b}=-\widehat{\Delta} f+2 f+\hat{\nabla}^{b}\left(P^{c} \hat{h}_{c b}\right) .
$$

As a result, we have

$$
\delta(V \widehat{H})=\sqrt{1+r^{2}}\left(-\frac{1}{2} \hat{h}^{a b}(\delta \hat{\sigma})_{a b}-\widehat{\Delta} f+2 f+\hat{\nabla}^{b}\left(P^{c} \hat{h}_{c b}\right)\right)+2 f+O\left(r^{6}\right)
$$

and

$$
\begin{aligned}
& \left.\frac{d}{d s}\right|_{s=0} \mathfrak{H}_{1} \\
= & \int \sqrt{1+r^{2}}\left(-\frac{1}{2} \hat{h}^{a b}(\delta \hat{\sigma})_{a b}-\widehat{\Delta} f+2 f+\hat{\nabla}^{b}\left(P^{c} \hat{h}_{c b}\right)\right)+2 f+\frac{1}{2} \hat{\sigma}^{a b}(\delta \hat{\sigma})_{a b} \sqrt{1+r^{2}} \widehat{H} d \hat{\Sigma}+O\left(r^{8}\right) \\
= & \int \sqrt{1+r^{2}} \frac{1}{2}\left(\widehat{H} \hat{\sigma}^{a b}-\hat{h}^{a b}\right)(\delta \hat{\sigma})_{a b}+4 f d \hat{\Sigma}+O\left(r^{8}\right)
\end{aligned}
$$


Rewriting the linearized isometric embedding equation in terms of $P^{a}$ and $f$, we have

$$
\hat{\nabla}_{a} P_{b}+\hat{\nabla}_{b} P_{a}+2 f \hat{h}_{a b}=(\delta \hat{\sigma})_{a b}
$$

Taking the trace and integrating, we get

$$
\int 2 f \widehat{H} d \hat{\Sigma}=\int \hat{\sigma}^{a b}(\delta \hat{\sigma})_{a b} d \hat{\Sigma}
$$

It follows that

$$
\int 4 f d \hat{\Sigma}=\int r \hat{\sigma}^{a b}(\delta \hat{\sigma})_{a b} d \hat{\Sigma}+O\left(r^{8}\right)
$$

and

$$
\begin{aligned}
\left.\frac{d}{d s}\right|_{s=0} \mathfrak{H}_{1} & =\int \sqrt{1+r^{2}} \frac{1}{2}\left(\widehat{H} \hat{\sigma}^{a b}-\hat{h}^{a b}\right)(\delta \hat{\sigma})_{a b}+r \hat{\sigma}^{a b}(\delta \hat{\sigma})_{a b} d \hat{\Sigma}+O\left(r^{8}\right) \\
& =\int \delta \tau\left(1+r^{2}\right)^{\frac{3}{2}} \hat{\nabla}_{a} \hat{\nabla}_{b} \tau \frac{1}{2}\left(\widehat{H} \hat{\sigma}^{a b}-\hat{h}^{a b}+r \hat{\sigma}^{a b}\right) d \hat{\Sigma}+O\left(r^{8}\right) \\
& =\int \delta \tau\left(1+r^{2}\right) \nabla_{a} \nabla_{b} Y^{0} \frac{1}{2}\left(\widehat{H} \hat{\sigma}^{a b}-\hat{h}^{a b}+r \hat{\sigma}^{a b}\right) d \hat{\Sigma}+O\left(r^{8}\right) .
\end{aligned}
$$

On the other hand, direct computation (see Theorem 5.2 of [13]) gives that

$$
\left.\frac{d}{d s}\right|_{s=0} \mathfrak{H}_{2}=\int\left(1+r^{2}\right) \delta \tau\left[\operatorname{div}\left(\left|H_{0}\right| \nabla Y^{0}\right)+\Delta \frac{\Delta Y^{0}}{\left|H_{0}\right|}-\operatorname{div}_{H_{0}}\right] .
$$

We conclude that

$$
\left.\operatorname{div} \alpha_{H_{0}}=\Delta \frac{\Delta Y^{0}}{\left|H_{0}\right|}+\operatorname{div}\left(\left|H_{0}\right| \nabla Y^{0}\right)-\frac{1}{2}\left(\left(\frac{1}{2}\left|H_{0}\right|+r\right) \sigma^{a b}-\stackrel{\circ}{A}^{a b}\right)\right) \nabla_{a} \nabla_{b} Y^{0}+O\left(r^{3}\right) .
$$

since

$$
\left.\frac{d}{d s}\right|_{s=0} \mathfrak{H}_{1}=\left.\frac{d}{d s}\right|_{s=0} \mathfrak{H}_{2},
$$

for any choice of $\delta \tau$

\section{Proposition 10.1.}

$$
\lim _{r \rightarrow 0}-r^{-5} \int_{\Sigma_{r}} \alpha_{H_{0}}\left(V^{2} \nabla \tau\right) d \Sigma_{r}=\frac{4}{3} A \int_{S^{2}} W_{0}^{2} d S^{2}-10 \frac{C_{i} C_{j}}{A} \int_{S^{2}} \tilde{X}^{i} W_{0} P_{j} d S^{2} .
$$

Proof. We compute 


$$
\begin{aligned}
& \int_{\Sigma_{r}} \alpha_{H_{0}}\left(V^{2} \nabla \tau\right) d \Sigma_{r} \\
= & \int_{\Sigma_{r}} \alpha_{H_{0}}\left[\left(A Y^{0}+C_{i} Y^{i}\right) \nabla Y^{4}+\left(A Y^{4}+B_{i} Y^{i}\right) \nabla Y^{0}+\left(C_{i} Y^{4}+B_{i} Y^{0}+D_{p} \epsilon_{p q i} Y^{q}\right) \nabla Y^{i}\right] d \Sigma_{r} \\
= & \int_{\Sigma_{r}} \alpha_{H_{0}}\left[A \nabla Y^{0}+\left(C_{i} Y^{4}+D_{p} \epsilon_{p q i} Y^{q}\right) \nabla Y^{i}\right] d \Sigma_{r}+O\left(r^{6}\right) \\
= & \int_{\Sigma_{r}} \alpha_{H_{0}}\left[A \nabla Y^{0}+\left(C_{i}\left(1+\frac{r^{2}}{2}\right)+D_{p} \epsilon_{p q i} Y^{q}\right) \nabla Y^{i}\right] d \Sigma_{r}+O\left(r^{6}\right) \\
= & \int_{\Sigma_{r}} \alpha_{H_{0}}\left[A \nabla Y^{0}+C_{i} \nabla Y^{i}\right] d \Sigma_{r}+O\left(r^{6}\right) .
\end{aligned}
$$

In the last equation, we used (10.1) and that $Y_{0}^{(3)}$ is perpendicular to $\tilde{X}^{i}$. Integrating by parts, we get

$$
-\int_{\Sigma_{r}} \alpha_{H_{0}}\left(V^{2} \nabla \tau\right) d \Sigma_{r}=\int_{\Sigma_{r}}\left(A Y^{0}+C_{i} Y^{i}\right) d i v \alpha_{H_{0}}+O\left(r^{6}\right)
$$

The proposition now follows from Proposition 8.1 of [13].

\section{Computing the Physical Hamiltonian}

In this section, we compute the limit of the third integral in equation (8.1):

$$
-\int_{\Sigma_{r}} \alpha_{H}\left(V^{2} \nabla \tau\right) d \Sigma_{r}
$$

\section{Proposition 11.1.}

$$
\lim _{r \rightarrow \infty}-r^{-5} \int_{\Sigma_{r}} \alpha_{H}\left(V^{2} \nabla \tau\right) d \Sigma_{r}=\int_{S^{2}}\left[\frac{4 A}{3} W_{0}^{2}+\frac{2}{3} C_{i} W_{i} W_{0}-\left(C_{i} \tilde{X}^{i}\right)|\beta|^{2}\right] d S^{2} .
$$

Proof. We compute

$$
\begin{aligned}
& \int_{\Sigma_{r}} \alpha_{H}\left(V^{2} \nabla \tau\right) d \Sigma_{r} \\
= & \int_{\Sigma_{r}}\left(\alpha_{H}\right)\left[A \nabla Y^{0}+\left(C_{i} Y^{4}+D_{p} \epsilon_{p q i} Y^{q}\right) \nabla Y^{i}\right] d \Sigma_{r}+O\left(r^{6}\right) \\
= & \int_{\Sigma_{r}}\left(\alpha_{H}\right)\left[A \nabla Y^{0}+\left(C_{i}\left(1+\frac{r^{2}}{2}\right)+D_{p} \epsilon_{p q i} Y^{q}\right) \nabla Y^{i}\right] d \Sigma_{r}+O\left(r^{6}\right)
\end{aligned}
$$

From Proposition 9.1 of [12], we have

$$
\int_{\Sigma_{r}}\left(\alpha_{H}\right)\left[A \nabla Y^{0}+C_{i} \nabla Y^{i}\right] d \Sigma_{r}=r^{5} \int_{S^{2}}\left[\frac{4 A}{3} W_{0}^{2}+\frac{2}{3} C_{i} W_{i} W_{0}-\left(C_{i} \tilde{X}^{i}\right)|\beta|^{2}\right] d S^{2}+O\left(r^{6}\right)
$$


As a result, it suffices to show that

$$
\int_{\Sigma_{r}}\left(\alpha_{H}\right)\left[\left(\frac{r^{2} C_{i}}{2}+D_{p} \epsilon_{p q i} Y^{q}\right) \nabla Y^{i}\right] d \Sigma_{r}=O\left(r^{6}\right)
$$

or simply that

$$
\begin{aligned}
\int_{S^{2}} \alpha_{H}^{(2)}\left(\tilde{\nabla} \tilde{X}^{i}\right) & =0 \\
\int_{S^{2}} \alpha_{H}^{(2)}\left(\epsilon_{p q i} \tilde{X} \tilde{X}^{q} \tilde{\nabla} \tilde{X}^{i}\right) & =0 \\
\int_{S^{2}} \alpha_{H}^{(3)}\left(\epsilon_{p q i} \tilde{X} \tilde{X}^{q} \tilde{\nabla} \tilde{X}^{i}\right) & =0
\end{aligned}
$$

The first integral vanishes due to Theorem 6.1 and that the spacetime is vacuum. For the other two integrals, we recall that

$$
\eta_{a}=\frac{r^{2}}{3} \beta_{a}+\frac{r^{3}}{4} D \beta_{a}+r^{4}\left[\frac{1}{10} D^{2} \beta_{a}-\frac{1}{45} \alpha_{a b} \beta^{b}\right]+O\left(r^{5}\right),
$$

$\alpha_{H}$ is the same as $\eta$ up to a gradient vector field and the rotation Killing field $\epsilon_{p q i} \tilde{X}^{q} \tilde{\nabla} \tilde{X}^{i}$ is divergence free. Hence, it suffice to show that

$$
\begin{gathered}
\int_{S^{2}} \beta\left(\epsilon_{p q i} \tilde{X}^{q} \tilde{\nabla} \tilde{X}^{i}\right)=0 \\
\int_{S^{2}} D \beta\left(\epsilon_{p q i} \tilde{X}^{q} \tilde{\nabla} \tilde{X}^{i}\right)=0 .
\end{gathered}
$$

From Lemma 4.7 and 4.9, we have

$$
\begin{gathered}
4 \int_{S^{2}} \beta\left(\epsilon_{p q i} \tilde{X}^{q} \tilde{\nabla} \tilde{X}^{i}\right)=\int_{S^{2}} \tilde{\nabla}^{a} \alpha_{a b}\left(\epsilon_{p q i} \tilde{X} \tilde{\nabla}^{q} \tilde{X}^{i}\right)=0 \\
5 \int_{S^{2}} D \beta\left(\epsilon_{p q i} \tilde{X}^{q} \tilde{\nabla} \tilde{X}^{i}\right)=\int_{S^{2}} \tilde{\nabla}^{a} D \alpha_{a b}\left(\epsilon_{p q i} \tilde{X} \tilde{\nabla}^{q} \tilde{X}^{i}\right)=0 .
\end{gathered}
$$

\section{Evaluating the Energy}

From Lemma 9.3. Proposition 9.1, 10.1 and 11.1 and Section 10 of [12], we conclude immediately that

$$
\lim _{r \rightarrow 0} r^{-5} E\left(\Sigma_{r}, Y_{r}\left(T_{0}\right), T_{0}\right)=\frac{1}{90}\left[Q\left(e_{0}, e_{0}, e_{0}, A e_{0}+C_{i} e_{i}\right)+\frac{\sum_{m, n} \bar{W}_{0 m 0 n}^{2}}{2 A}\right] .
$$

To minimize $E\left(\Sigma_{r}, Y_{r}\left(T_{0}\right), T_{0}\right)$ among choices of $T_{0}$, let

$$
E\left(\Sigma_{r}, Y_{r}\left(T_{0}\right), T_{0}\right)=E\left(\Sigma, Y\left(T_{0}\right), T_{0}\right)^{(5)} r^{5}+O\left(r^{6}\right) .
$$

We consider the following vector

$$
U=\left(\frac{1}{2} \sum \bar{W}_{0 k m n}^{2}+\sum \bar{W}_{0 m 0 n}^{2}, 2 \sum \bar{W}_{0 m 0 n} \bar{W}_{0 m i n}\right) .
$$


$U$ is future directed non-spacelike. It is timelike unless the Weyl curvature is of the form given by [10, Lemma 11.2].

It is easy to see that the energy functional $E\left(\Sigma, Y\left(T_{0}\right), T_{0}\right)^{(5)}$ is non-negative. Moreover, it is positive and proper when $U$ is timelike. Hence, when $U$ is timelike, there is at least one choice of $T_{0}=\left(A, C_{i}\right)$ which minimizes $E\left(\Sigma, Y\left(T_{0}\right), T_{0}\right)^{(5)}$. We show that under the same condition, the minimizer is unique.

Lemma 12.1. If $V$ is timelike, there is a unique $\left(A, C_{i}\right)$ that minimizes $E\left(\Sigma, X\left(T_{0}\right), T_{0}\right)^{(5)}$.

Proof. From the remark after Proposition 2.1, we have

$$
A \geq \sqrt{1+|\vec{C}|^{2}} \geq 1
$$

Moreover,

where

$$
E\left(\Sigma, Y\left(T_{0}\right), T_{0}\right)^{(5)}=\frac{1}{90}\left[Q\left(e_{0}, e_{0}, e_{0}, A e_{0}+C_{i} e_{i}\right)+\frac{\sum_{m, n} \bar{W}_{0 m 0 n}^{2}}{2 A}\right]
$$

$$
Q\left(e_{0}, e_{0}, e_{0}, A e_{0}+C_{i} e_{i}\right)=\left(\frac{1}{2} \sum_{k, m, n} \bar{W}_{0 k m n}^{2}+\sum_{m, n} \bar{W}_{0 m 0 n}^{2}\right) A+2 \sum_{m, n, i} \bar{W}_{0 m 0 n} \bar{W}_{0 m i n} C_{i} .
$$

Hence, fixing $\vec{C}, E\left(\Sigma, Y\left(T_{0}\right), T_{0}\right)^{(5)}$ is increasing in $A$ and the minimum can only occur on the set of observers, $O$, such that

$$
A=\sqrt{1+|\vec{C}|^{2}}
$$

From the proof of [10, Lemma 11.3], $E\left(\Sigma, Y\left(T_{0}\right), T_{0}\right)^{(5)}$ is a strictly convex function of $\left(C_{1}, C_{2}, C_{3}\right)$ on $O$. This finishes the proof of the lemma

As a result, we have the following theorem for the small sphere limit of the quasi-local energy in vacuum spacetimes with reference in the AdS space:

Theorem 12.1. Let $\Sigma_{r}$ be the family of spheres approaching $p$ constructed in Section 4.

(1) For each observer $T_{0}$ in the AdS space, there is a pair $\left(Y_{r}\left(T_{0}\right), T_{0}\right)$ solving the leading order term of the optimal embedding equation of $\Sigma_{r}$ (see Lemma 6.1 and 8.2). For this pair $\left(Y_{r}\left(T_{0}\right), T_{0}\right)$, we have

$$
\lim _{r \rightarrow 0} r^{-5} E\left(\Sigma_{r}, Y_{r}\left(T_{0}\right), T_{0}\right)=\frac{1}{90}\left[Q\left(e_{0}, e_{0}, e_{0}, A e_{0}+C_{i} e_{i}\right)+\frac{\sum_{m, n} \bar{W}_{0 m 0 n}^{2}}{2 A}\right] .
$$

(2) Suppose $Q\left(e_{0}, e_{0}, e_{0}, \cdot\right)$ is dual to a timelike vector. Let $\mathcal{P}$ denote the set of $\left(Y, T_{0}\right)$ admitting a power series expansion given in equation (6.1). We have

$$
\inf _{\left(Y, T_{0}\right) \in \mathcal{P}} \lim _{r \rightarrow 0} r^{-5} E\left(\Sigma_{r}, Y, T_{0}\right)=\inf _{\left(A, C_{i}\right) \in \mathbb{H}^{3}} \frac{1}{90}\left[Q\left(e_{0}, e_{0}, e_{0}, A e_{0}+C_{i} e_{i}\right)+\frac{\sum_{m, n} \bar{W}_{0 m 0 n}^{2}}{2 A}\right] .
$$

where $\mathbb{H}^{3}$ denotes the set of unit timelike future directed vector in $\mathbb{R}^{3,1}$. The infimum is achieved by a unique $\left(A, C_{i}\right) \in \mathbb{H}^{3}$. 


\section{REFERENCES}

[1] L. F. Abbott and S. Deser, Stability of gravity with a cosmological constant, Nuclear Phys. B 195 (1982), no. 1, 76-96.

[2] A. Ashtekar and A. Magnon, Asymptotically anti-de Sitter space-times, Classical Quantum Gravity 1 (1984), no. 4, L39-L44. I

[3] L. Bel, Introduction d'un tenseur du quatrième ordre, C. R. Acad. Sci. Paris 248 (1959), 1297-1300.

[4] L. Bieri and N. Zipser, Extensions of the stability theorem of the Minkowski space in general relativity, AMS/IP Studies in Advanced Mathematics, 45, Amer. Math. Soc., Providence, RI, 2009.

[5] J. D. Brown, S. R. Lau, and J. W. York, Jr., Energy of isolated systems at retarded times as the null limit of quasi-local energy, Phys. Rev. D (3) $5 \mathbf{5}$ (1997), no. 4, 1977-1984.

[6] J. D. Brown, S. R. Lau, and J. W. York, Jr., Canonical quasi-local energy and small spheres. Phys. Rev. D (3) 59 (1999), no. 6, 064028.

[7] C. Cederbaum, J. Cortier and A. Sakovich, On the center of mass of asymptotically hyperbolic initial data sets, Ann. Henri Poincaré 17 (2016), no. 6, 1505-1528.

[8] P-N. Chen, P.-K. Hung, M.-T. Wang and S.-T. Yau, The rest mass of an asymptotically Anti-de Sitter spacetime, To appear in Ann. Henri Poincare

[9] P.-N. Chen, M.-T. Wang and Y.-K. Wang, Rigidity of time-flat surfaces in the Minkowski spacetime, Math. Res. Lett. 21 (2014), no. 6, 1227-1240.

[10] P.-N. Chen, M.-T. Wang and S.-T. Yau, Evaluating quasi-local energy and solving optimal embedding equation at null infinity, Comm. Math. Phys. 308 (2011), no.3, 845-863.

[11] P.-N. Chen, M.-T. Wang and S.-T. Yau, Minimizing properties of critical points of quasi-local energy, Comm. Math. Phys. 329 (2014), no. 3, 919-935.

[12] P-N. Chen, M.-T. Wang and S.-T. Yau, Evaluating small sphere limit of the Wang-Yau quasi-local energy, To appear in Comm. Math. Phys.

[13] P-N. Chen, M.-T. Wang and S.-T. Yau, Quasi-local energy with respect to de Sitter/anti-de Sitter reference, arXiv:1603.02975.

[14] D. Christodoulou and S. Klainerman, The global nonlinear stability of the Minkowski space. Princeton Mathematical Series, 41. Princeton University Press, Princeton, NJ, 1993.

[15] D. Christodoulou and S.-T. Yau, Some remarks on the quasi-local mass, in Mathematics and general relativity (Santa Cruz, CA, 1986), 9-14, Contemp. Math., 71, Amer. Math. Soc., Providence, RI.

[16] P. T. Chruściel and M. Herzlich, The mass of asymptotically hyperbolic Riemannian manifolds, Pacific J. Math. 212 (2003), no. 2, 231-264.

[17] P. T. Chruściel and G. Nagy, The Hamiltonian mass of asymptotically anti-de Sitter space-times, Classical Quantum Gravity 18 (2001), no. 9, L61-L68.

[18] X.-Q. Fan, Y. Shi, and L.-F. Tam, Large-sphere and small-sphere limits of the Brown-York mass. Comm. Anal. Geom. 17 (2009), no. 1, 37-72.

[19] R. Geroch, Energy extraction, Annals of the New York Academy of Sciences 224.1 (1973): 108-117.

[20] G. W. Gibbons, C. M. Hull, and N. P. Warner, The stability of gauged supergravity, Nuclear Phys. B 218 (1983), no. 1, 173-190.

[21] M. Henneaux and C. Teitelboim, Asymptotically anti-de Sitter spaces, Comm. Math. Phys. 98 (1985), no. 3, 391-424.

[22] G. T. Horowitz and B. G. Schmidt, Note on gravitational energy, Proc. Roy. Soc. London Ser. A 381 (1982), no. 1780, 215-224.

[23] G. Huisken and T. Ilmanen, The inverse mean curvature flow and the Riemannian Penrose inequality, J. Differential Geom. 59 (2001), no. 3, 353-437.

[24] K.-K. Kwong and L.-F. Tam, Limit of quasilocal mass integrals in asymptotically hyperbolic manifolds, Proc. Amer. Math. Soc. 141 (2013), no. 1, 313-324.

[25] C.-Y. Lin and Y.-K. Wang, On isometric embeddings into anti-de Sitter spacetimes, Int. Math. Res. Not. IMRN 2015, no. 16, 7130-7161. 
[26] P. Miao and L.-F. Tam, On second variation of Wang-Yau quasi-local energy, Ann. Henri Poincaré 15 (2014), no. 7, 1367-1402.

[27] P. Miao, L.-F. Tam and N. Xie, Quasi-local mass integrals and the total mass, J. Geom. Anal. 27 (2017), no. 2, 1323-1354.

[28] L. B. Szabados, Quasi-local energy-momentum and angular momentum in general relativity, Living Rev. Relativity, 12 (2009), no. 4.

[29] R. Penrose, Quasilocal mass and angular momentum in general relativity, Proc. Roy. Soc. London Ser. A 381 (1982), no. 1780, 53-63.

[30] M.-T. Wang and S.-T. Yau, Isometric embeddings into the Minkowski space and new quasi-local mass, Comm. Math. Phys. 288 (2009), no. 3, 919-942.

[31] M.-T. Wang and S.-T. Yau, Limit of quasilocal mass at spatial infinity, Comm. Math. Phys. 296 (2010), no. 1, 271-283.

[32] D. Wiygul The Bartnik-Bray outer mass of small metric spheres in time-symmetric 3-slices, arXiv:1609.09460

[33] P. P. Yu, The limiting behavior of the Liu-Yau quasi-local energy, arXiv:0706.1081v1. 